\title{
Drosophila tumor suppressor PTEN controls cell size and number by antagonizing the Chico/PI3-kinase signaling pathway
}

\author{
Deborah C.I. Goberdhan, ${ }^{1}$ Nuria Paricio, ${ }^{2}$ Emma C. Goodman, ${ }^{1}$ Marek Mlodzik, ${ }^{2}$ \\ and Clive Wilson ${ }^{1,3}$ \begin{abstract}
Kingdom; ${ }^{2}$ Developmental Biology Programme, European Molecular Biology Laboratory (EMBL),
Heidelberg, D69117, Germany
\end{abstract} \\ ${ }^{1}$ Research School of Biosciences, Department of Biosciences, University of Kent, Canterbury, Kent CT2 7NJ, United
}

\begin{abstract}
The human tumor suppressor gene PTEN encodes a putative cytoskeleton-associated molecule with both protein phosphatase and phosphatidylinositol 3,4,5-trisphosphate (PIP3) 3-phosphatase activities. In cell culture, the lipid phosphatase activity of this protein is involved in regulating cell proliferation and survival, but the mechanism by which PTEN inhibits tumorigenesis in vivo is not fully established. Here we show that the highly evolutionarily conserved Drosophila PTEN homolog, DPTEN, suppresses hyperplastic growth in flies by reducing cell size and number. We demonstrate that DPTEN modulates tissue mass by acting antagonistically to the Drosophila Class I phosphatidylinositol 3-kinase, Dp110, and its upstream activator Chico, an insulin receptor substrate homolog. Surprisingly, although DPTEN does not generally affect cell fate determination, it does appear to regulate the subcellular organization of the actin cytoskeleton in multiple cell types. From these data, we propose that DPTEN has a complex role in regulating tissue and body size. It acts in opposition to Dp110 to control cell number and growth, while coordinately influencing events at the cell periphery via its effects on the actin cytoskeleton.
\end{abstract}

[Key Words: Cell proliferation; growth control; actin cytoskeleton; insulin signaling; PI3-kinase; Akt/PKB]

Received August 20, 1999; revised version accepted October 26, 1999.

The human tumor suppressor gene PTEN (phosphatase and tensin homolog on chromosome 10; Li et al. 1997a), which is also known as MMAC1 (Steck et al. 1997) or TEP1 (Li and Sun 1997), has been implicated in a diverse range of tumors (for review, see Myers and Tonks 1997; Smith and Ashworth 1998). In fact, it is the most commonly mutated gene in some forms of malignancy, such as glioma (Wang et al. 1997). PTEN is also linked to two dominantly inherited disorders-Cowden Disease and Bannayan-Zonana Syndrome (CD and BZS, respectively; Marsh et al. 1998), which lead to multiple defects, including excessive developmental growth of specific structures such as digits, the formation of many benign outgrowths known as hamartomas, and an increased occurrence of cancer.

The PTEN protein contains a protein phosphatase domain with similarity to dual specificity phosphatases (Li et al. 1997a; Li and Sun 1997; Steck et al. 1997). This is

${ }^{3}$ Corresponding author.

E-MAIL C.Wilson@ukc.ac.uk; FAX 01227763912. embedded within a domain with homology to an actinbinding motif in the focal adhesion-associated protein tensin. Immunohistochemical evidence has suggested that like tensin, PTEN is associated with the cytoskeleton (Li and Sun 1997).

In vitro, PTEN can dephosphorylate acidic polypeptide substrates (Myers et al. 1997), a key component of focal adhesions called focal adhesion kinase (FAK; Tamura et al. 1998) and the SH2-phosphotyrosine adaptor protein Shc (Gu et al. 1999). It has recently been shown, however, that PTEN also has phosphoinositide 3-phosphatase activity (Maehama and Dixon 1998; Myers et al. 1998), which catalyzes the interconversion of two key lipid messengers in the cell, phosphatidylinositol 3,4,5trisphosphate (PIP3) and phosphatidylinositol 4,5-bisphosphate (PIP2; Leevers et al. 1999). A missense mutation in PTEN, G129E, which has been linked to tumorigenesis, appears to disrupt only the lipid phosphatase activity of this molecule (Furnari et al. 1998; Myers et al. 1998), suggesting that this activity has an important role in growth control.

Several groups have generated PTEN knockout mice 
and shown that they die during embryogenesis (Di Cristofano et al. 1998; Stambolic et al. 1998; Suzuki et al. 1998). The precise developmental defects in these embryos have not been characterized, but tissue growth appears to be affected (Stambolic et al. 1998). Studies in cell culture have indicated that PTEN can control cell number by regulating both cell proliferation and survival. In fibroblasts cultured from PTEN mutant mice, protein kinase $\mathrm{B}$ (PKB or Akt), a downstream effector of PIP3, is hyperactivated and promotes cell survival (Stambolic et al. 1998). This indicates that PTEN's lipid phosphatase activity has an important role in regulating cell number. Furthermore, overexpression of wild-type and mutant PTEN proteins in glioma cells has demonstrated that the lipid phosphatase activity of this molecule can produce a serum-sensitive cell cycle arrest in $G_{1}$ phase, thereby suppressing cell proliferation (Furnari et al. 1998). Other experiments in cell culture, however, have implicated the protein phosphatase activity of PTEN in some of its biological functions. In particular, overexpression of wildtype PTEN and mutant PTEN lacking lipid phosphatase activity can reduce levels of FAK phosphorylation and the formation of focal adhesions, thereby inhibiting cell migration and invasiveness (Tamura et al. 1998). Therefore, the antitumorigenic effects of PTEN in vivo may involve both its lipid and protein phosphatase activities, but the relative contributions of each activity remain unclear.

Homologs of PTEN have been identified in both yeast (Li et al. 1997b) and the nematode Caenorhabditis elegans (Ogg and Ruvkun 1998). In nematodes, genetic studies have demonstrated that the daf-18 gene encodes a PTEN homolog that regulates metabolism and developmental timing via its lipid phosphatase activity. daf18 , however, does not appear to affect tissue growth significantly, limiting the usefulness of nematodes as a model system to study the antitumorigenic functions of PTEN in vivo.

Here we describe the cloning and genetic characterization of a highly evolutionarily conserved Drosophila homolog of PTEN, DPTEN. We show that DPTEN not only controls cell number in flies, but also affects the size of cells and appears to have a general role in regulating the subcellular organization of the actin cytoskeleton. Although the mechanism by which DPTEN modulates the cytoskeleton remains unclear, the effects on cell size and number are antagonized by the Drosophila class I PI3kinase homolog Dp110 (Leevers et al. 1996), the enzyme that converts PIP2 into PIP3, suggesting that the lipid phosphatase activity of DPTEN is involved in growth control. Loss of DPTEN function completely suppresses the severe reduction in tissue growth caused by mutations in chico, which encodes a component of the insulin receptor complex that activates Dp110 and regulates body size (Böhni et al. 1999). Our results indicate that the effects on growth in DPTEN mutant tissue are coordinated with changes in the actin cytoskeleton, which may be important in controlling the spreading of hyperplastic mutant cells.

\section{Results \\ Identification of Drosophila PTEN}

We have analyzed two Drosophila genes previously, Dror, encoding a neural-specific receptor tyrosine kinase (Wilson et al. 1993), and basket, encoding the homolog of c-Jun amino-terminal kinase (Sluss et al. 1996; RiesgoEscovar et al. 1996), which map adjacent to each other at $31 \mathrm{~B} / \mathrm{C}$ on the second chromosome (Fig. 1A). Several deficiency chromosomes were characterized that uncover one or both of these genes and also affect the adjacent gene chico, which codes for a homolog of mammalian insulin receptor substrates, IRS1-4 (Böhni et al. 1999). One of these deficiencies, $D f(2 L) 170 B$, was of particular interest because it deletes sequences proximal to Dror and produced an overgrowth phenotype in homozygous clones. This effect was not observed with deficiencies $[D f(2 L) 41 C$ and $D f(2 L) 147 F]$ that only delete Dror, $D J N K$, and chico (Fig. 1A). Clones generated with these smaller deficiencies contain reduced numbers of small cells attributable to the loss of chico function (Böhni et al. 1999; data not shown). During a chemical mutagenesis screen using the $D f(2 L) 170 B$ chromosome, we identified a new lethal complementation group that maps proximal to Dror and affects tissue growth. Sequence analysis of genomic and cDNA clones revealed a novel gene at this locus encoding the Drosophila PTEN homolog, DPTEN (Figs. 1B,2).

The DPTEN transcription unit is just over $4 \mathrm{~kb}$ in length, contains 11 introns and produces a transcript of $2.2 \mathrm{~kb}$, which encodes a conceptual protein of 509 amino acids. Upstream of the putative translation start site, there is at least one stop codon in every reading frame. Sequence analysis of multiple cDNAs and comparison with the DPTEN genomic sequence revealed two notable features (Fig. 1B). First, some cDNAs contain a 9-bp deletion at genomic position 3001 that removes three amino acids from the encoded protein; a cDNA with this deletion (clone $5 \mathrm{C}$ ) has rescue activity (see below), and the deletion has also been identified in genomic DNA from at least one Drosophila strain /data not shown), indicating it is a polymorphism. Second, there are two alternatively spliced DPTEN mRNAs, producing proteins differing by a few amino acids at their carboxyl termini (see Fig. 1B). We have used a putative full-length cDNA, which includes exon 11 sequences and therefore encodes the shorter form of DPTEN, for subsequent rescue experiments.

The predicted DPTEN protein was aligned and compared with other known PTEN homologs. The aminoterminal half of the DPTEN protein has been particularly highly conserved over evolution, sharing 65\% identity with human PTEN in this region (Fig. 2; Li et al. 1997a; Steck et al. 1997) and $\sim 40 \%$ and 30\% identity, respectively, with the more divergent Caenorhabditis elegans (Ogg and Ruvkun 1998) and yeast (Li et al. 1997b) PTEN homologs. Like its counterparts in other organisms, DPTEN contains a putative protein phosphatase motif within a larger region related to an actin binding domain in the cytoskeletal protein tensin. A number of 
Goberdhan et al.
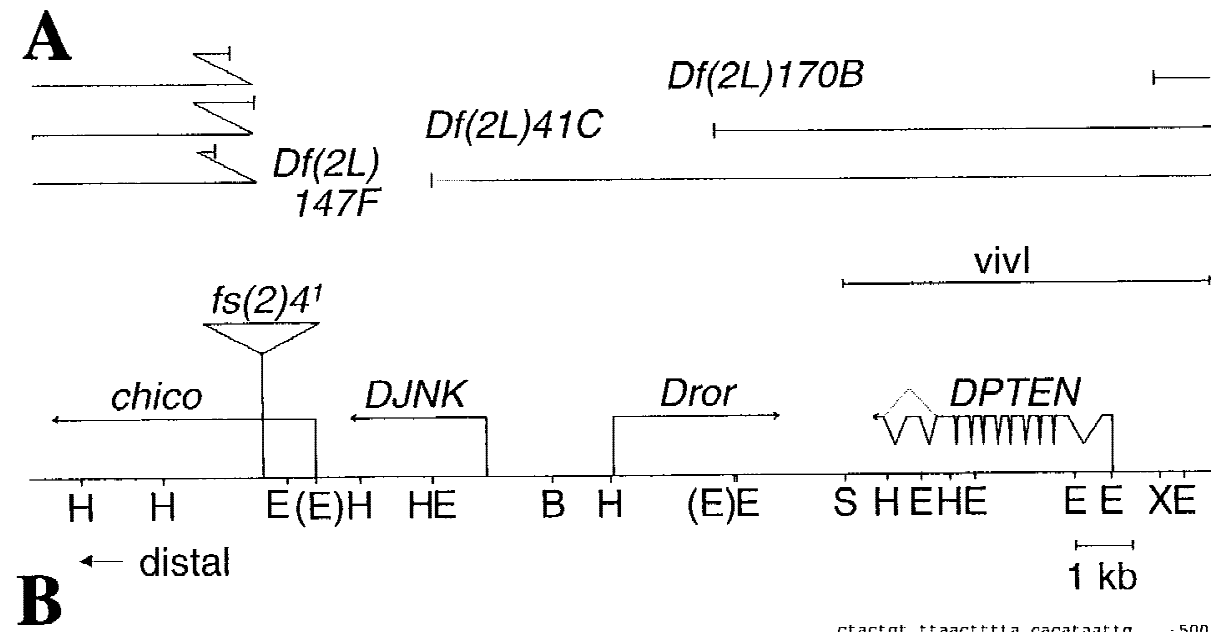

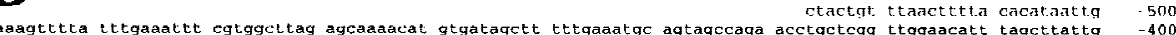
acttaaacat acccatatgc acatacatac atatgtatta accagtgggc acatatatre gtgtggtgct gatagtgtaa atgtgtaaaa tgtetgtgga attgtaaata tgtttttgtt taacaaata tgtacataaa tgcacaaaa teggtgcata tatgtgcaca tgcccatcat ccatatctgc atattectgt -200 acatgcatat gtgctctaac tacatacat accaccatte geaaacagtt tgtcaaaaa gtgtgtctac teggcgagat aaaacaaaa acagttgcca -100 aatcagtcge tatgttaagt aattcgaaat tactgctegg tgtttctaad acggattttg attttgatte tcaccecteg aaaategcee cetcageca CAT'STGACAA CCAATCTGAA TGTATTATGC CAAGCGGAAG ATTGTTATAT TGTTTATCTT TGATAATATG CCGATACATg taagtatgte tCaCgtgtgL 200 gecgcaaatg cctgcctgtg tgtgtgtgtg tgtgtgtgtg tgtgtgtatg tacatatgtg cgtgtgcatg tgegagtgtg tgtgagtgtg tgeatgcttg 300 tgtctgtatg tgtatgtacg tgcttaata tgtatccaag caagaactgc azacaggaat atatcttagc cqagtaagaa grattagttt atatagaces 500 acctaagtgg atcgggagaa aacaggccea ttatattagt tccatatatc ccttragaat gccttggcac agtataatta agcagtttct attgaccata 000 ttagaaett gtctgctogtt asaggattta ggtttgaatt cgcagtagaa aactaagctg aattgagtca atacttttet aacttgcrgt ggaaaatasa 800 cagattagtt agaatcatat gcatattat taatatgctt tacattcet teagaacgta cactttgcaa tggagtaat agAAGACAAG CACTGGTTCC 900 CAAATACATT TGAATGGATG CAGCTAATCT AATTTAAATA AAAAAGACAA GTCTTTAAAC CTTAAAAATA TGGCCAACAC TATTTCGTTA ATGTCCAACG 1000

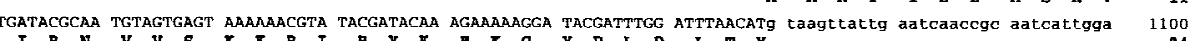

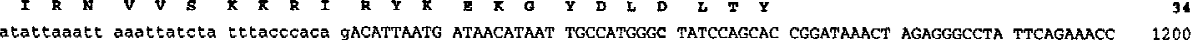
$I$ N D M I I A M G Y P A

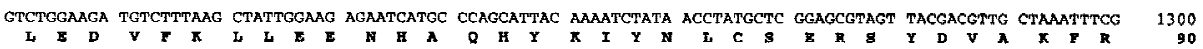
GGGgtante ttgatgaacc ttttctgttt gtcggcgtaa aatatgtaa atatactatt tcgcattaca gAGAGTAGCT GTTTATCCGT TCGATGATCA $G$ a

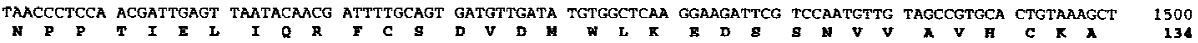

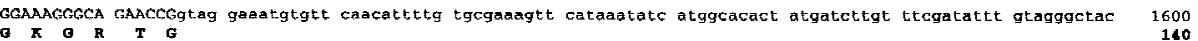

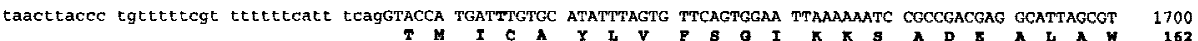
GGTATGATGA GAAGCGTACA AAGGACCGAA AAGGTGTGAC AATCCCATCT CAGCGTCGAT ATGTTCAGTA TTTTTCCAAA CTAGTTTGTT CAAGTGTTCC 1800 ATATTCGAAA GTAAGCCTPA ACgtgagtat ttccagcage agcgccacat tcagcgccat acaaaatcta aatatgcatt ttttgtatct cagGTATGTG 1900

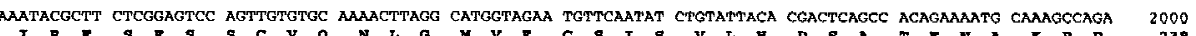
Tgtaagtata taaagtatat agatacatca ttaaaaatgt atatttcaca tabttcagt gttcettttc gaataccttt ctaaccagna gtaatattct 2100

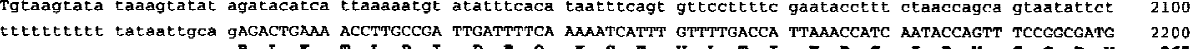
TAAAATTTGA ACTCACAAAA AAATCCCCAG ATAAAATCAT TTGTCACTT TGGCTAAATA CGTTTTTTGT ACGAAACTAT TCACgtaagt atact tcgcacatgc attaatasa cgatttaatt aacattetc tttagCTTGC GAGTCCGATG GAACAGTTAA CAAATATATA CACACTTTAA GCAAATCAGA 2400

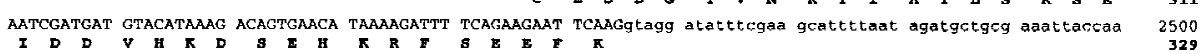
atatatgtge ttgctctett etcagATTTC AaTCGTTTTT GAGGCAGAGA ATTTTAGCAA TGATGTTCAA GCTGAAGCGT CCCAAAAAGA GACAAACGAA 2600

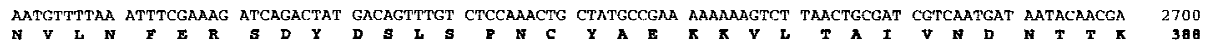
AAAGTCAAAC AATAGAAACG TTGGATCATA AGGATATAGT Tgtaggtgaa aacacgt tet gcgcagatca atatgct taa tetataaate taatattegt 2800

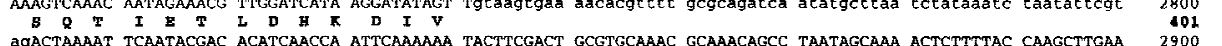

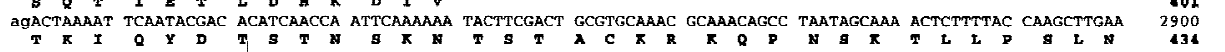

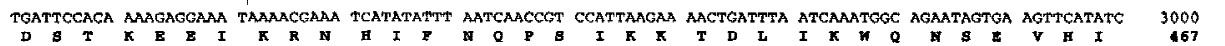

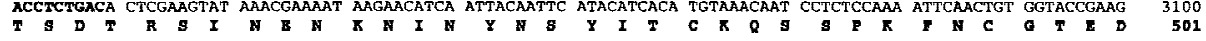

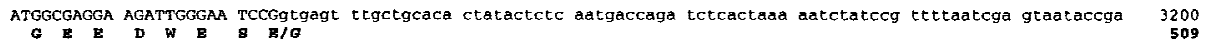
acaattgttc taattcaca tttatttaat actaagagtt tegattcaat tgatttctgg accattatog gtttgatttt gtaattaatt gtattgtatg 3300 agtattcaaa trạagatttg ttanaagaga tagcctttat cgacatatgc tatcgtccac acatttatat tcttattgac trattgaate cgccagaATA 3400

ATCACCCGAG ACGATTTAAA AGACTTCTGG CIGAACGATC TCTTAATTTT GATGTATAAA ACGAGGCCAC CAGAACTGGA TTTTATATGTC CCCAAGACTT 3500 TTACGAGTAT ACTAGCAGAG ACAGGCTTAC ACAGATCCAC TTCCTTTCAC GGGGAACCGA TCGATTACAG CTGCGCTCTG CACATCTGTT TAGaATTtAa 3600 TGTACATAGg tgagtgagtg ggagcccatg cctgeggetc gaatggtcaa ctaaccggca atcettaate agtatecges tegatttea teacacatec 3700

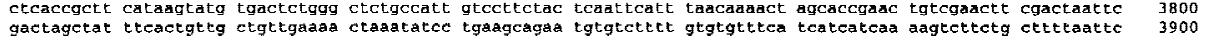
gactagetat eteacgttg etgttgaaa ctaaatatce tgaggeagaa tgtgtettet gtgtgtttea teaceateaa aagtettetg ctttraate 3900 etteteret tecagGTGAA TCAACATACC TGTAATCAAT GCTTATTACG GACTTATAAT TCTTGGTGAT DFAGCAAAGT RATTAAAATT GCTAAGTAAT 4100

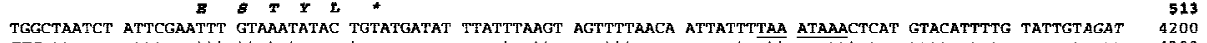

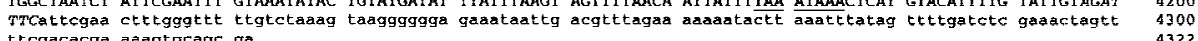
ttcgacacga aaagtgeagc ga 
amino acids shared by all members of the PTEN family are not present in other protein phosphatases or tensin family members (Fig. 2). These residues may have an important role in the specific functions of PTEN, such as its phosphoinositide 3-phosphatase activity. Sequence comparison of the carboxy-terminal half of DPTEN with human PTEN also highlights a number of conserved regions (Fig. 2), which may be involved in the regulation of this molecule. Drosophila should therefore provide a powerful in vivo model to study PTEN function, particularly because nearly all the residues that are known to be affected by missense mutations in cancer or PTENlinked dominant genetic disorders in humans (Maehama and Dixon 1999) are conserved in the DPTEN protein (Fig. 2).

To confirm that the DPTEN gene is allelic to the novel complementation group we had identified, constructs containing a genomic copy of DPTEN (Fig. 1A) and a DPTEN cDNA under the control of the hsp 70 promoter were introduced into flies and tested for rescue activity. Flies transheterozygous for $D f(2 L) 170 B$ and any of three different alleles in the complementation group die as late embryos or early first-instar larvae. This lethality was rescued by both the genomic construct and by the noninduced expression of the hs-DPTEN cDNA construct at $25^{\circ} \mathrm{C}$, thereby demonstrating that the mutations are DPTEN alleles.

\section{DPTEN regulates cell number and size and affects assembly of specific cytoskeleton-dependent structures}

Because animals transheterozygous for strong DPTEN alleles die with no obvious phenotypes, we investigated the functions of this gene further by generating homozygous mutant clones in heterozygous animals using the FLP/FRT system (Xu and Rubin 1993). We found that two DPTEN alleles, DPTEN ${ }^{1}$ and DPTEN ${ }^{3}$, produced growth phenotypes slightly more severe than those gen- erated by $D f(2 L) 170 B$, the chromosomal deficiency deleting DPTEN, Dror, DINK, and chico (Fig. 1A). DPTEN ${ }^{1}$ and $D P T E N^{3}$ also behaved similarly to the deficiency in combination with a weak, nonlethal DPTEN mutation (D. Goberdhan and C. Wilson, unpubl.), suggesting that they are strong or null DPTEN alleles.

The adult compound eye in Drosophila is composed of 700 regularly arranged unit eyes or ommatidia, each containing the same complement of photoreceptors and accessory cells. Mutant clones can be generated in the eye and other tissues by somatic recombination of homologous chromosomes in heterozygous animals. If only the nonmutant chromosome and not the mutant chromosome is marked with a copy of the white $\left(w^{+}\right)$gene, mutant clones can be distinguished by the absence of pigment. The wild-type twin spot resulting from a recombination event with these chromosomes contains increased levels of pigment relative to the remainder of the eye, as the cells in this region carry two copies of the $W^{+}$gene. Ommatidia within clones homozygous for $D P T E N^{1}, D P T E N^{3}$, or $D f(2 L) 170 B$ were enlarged and bulged from the surface of the eye (for examples, see Figs. $3, \mathrm{~A}, \mathrm{~B}, \mathrm{D}$, and 4, A-C). Ommatidial facets were occasionally fused, and there were frequently clusters of morphologically abnormal interommatidial bristles or clustered sockets in mutant regions, particularly (but not exclusively) in the dorsal half of the eye (Fig. 3D-F). To assess the effect of loss of DPTEN function on the regulation of cell number, we compared the numbers of ommatidia in multiple mutant clones and their wild-type twin spots. Mutant clones typically contained at least twice as many ommatidia as neighboring twin spots [the ratio of ommatidia in mutant versus wild-type tissue was $2.72 \pm 0.95(n=58)$; e.g., see Fig. $3 \mathrm{~A}]$, suggesting that mutant cells overproliferate, a result supported by analysis of clones at larval stages (see below; e.g., Fig. 7C, below). The largest DPTEN clones included more than half of the adult eye and formed a hyperplastic tumor-like overgrowth (Fig. 3C). This hyperplastic phenotype, however,

\footnotetext{
Figure 1. Molecular characterization of DPTEN. (A) Chromosomal location of DPTEN. The DPTEN gene resides at 31B/C on the second chromosome in a locus including the genes Dror (Wilson et al. 1993), bsk/DINK (Riesgo-Escovar 1996; Sluss et al. 1996), and chico (Böhni et al. 1999). For our analysis, we employed three deficiencies generated by imprecise excision of a P element that disrupts chico [ $f_{S}(2) 4^{1}$; see Sluss et al. 1996; Böhni et al. 1999]. $D f(2 L) 41 C$ deletes the $5^{\prime}$ half of the Dror gene; embryos homozygous for this deficiency fail to express Dror transcripts (data not shown), indicating that it is null for Dror. EMS-induced mutations in DPTEN map proximal to Dror, as they fail to complement the largest deficiency, $D f(2 L) 170 B$, but complement $D f(2 L) 41 C$ and $D f(2 L) 147 F$. Figure also shows the extent of a genomic rescue construct, vivI, used in this study. Restriction enzyme sites: BamHI (B); EcoRI (E); HindIII $(\mathrm{H})$; SaII (S); XbaI (X). (B) Sequence of the DPTEN genomic locus. Comparison of multiple DPTEN cDNA clones indicates that the DPTEN gene encodes a transcript of at least $2.2 \mathrm{~kb}$ (uppercase nucleotides), containing an ORF of 509 amino acids (in bold below the nucleotide sequence; stop codons are represented by asterisks). The first nucleotide of the longest available cDNA is designated nucleotide one. The transcription unit is interrupted by 11 introns (lowercase nucleotides), all including the consensus sequences for 5' donor and 3' acceptor splice junctions (Mount 1982). Two methionine residues are found within the first eight residues of the ORF. The amino-terminal methionine, which is preceded by stop codons (underscored) in all three reading frames, is likely to be the translation initiator, because it matches the translation start consensus ([C/A]AA[C/A]ATG; Cavener 1987), except that an additional T has been inserted directly $5^{\prime}$ of the ATG, a feature also found in the eyeless gene (Quiring et al. 1994). The consensus polyadenylation signal site (position 4168; underscored) is located 30 bases upstream of the polyA tail (which in different cDNAs is located either at position 4197 or 4203; additional bases in extended transcript are in italics). Some cDNA and genomic sequences have a polymorphic deletion; they lack nine nucleotides from positions 3001 to 3009 (bold). All other polymorphisms do not affect the encoded DPTEN protein; they are also shown in bold (see also Materials and Methods). One cDNA (clone 6C) is derived from an alternatively spliced mRNA (dotted line in Fig. 1A; creating a splice fusion between nucleotides 3124 and 4016) that lacks exon 11 sequences (nucleotides 3397-3609) and therefore encodes a protein containing an additional 5 amino acids (italicized) at its carboxyl terminus.
} 


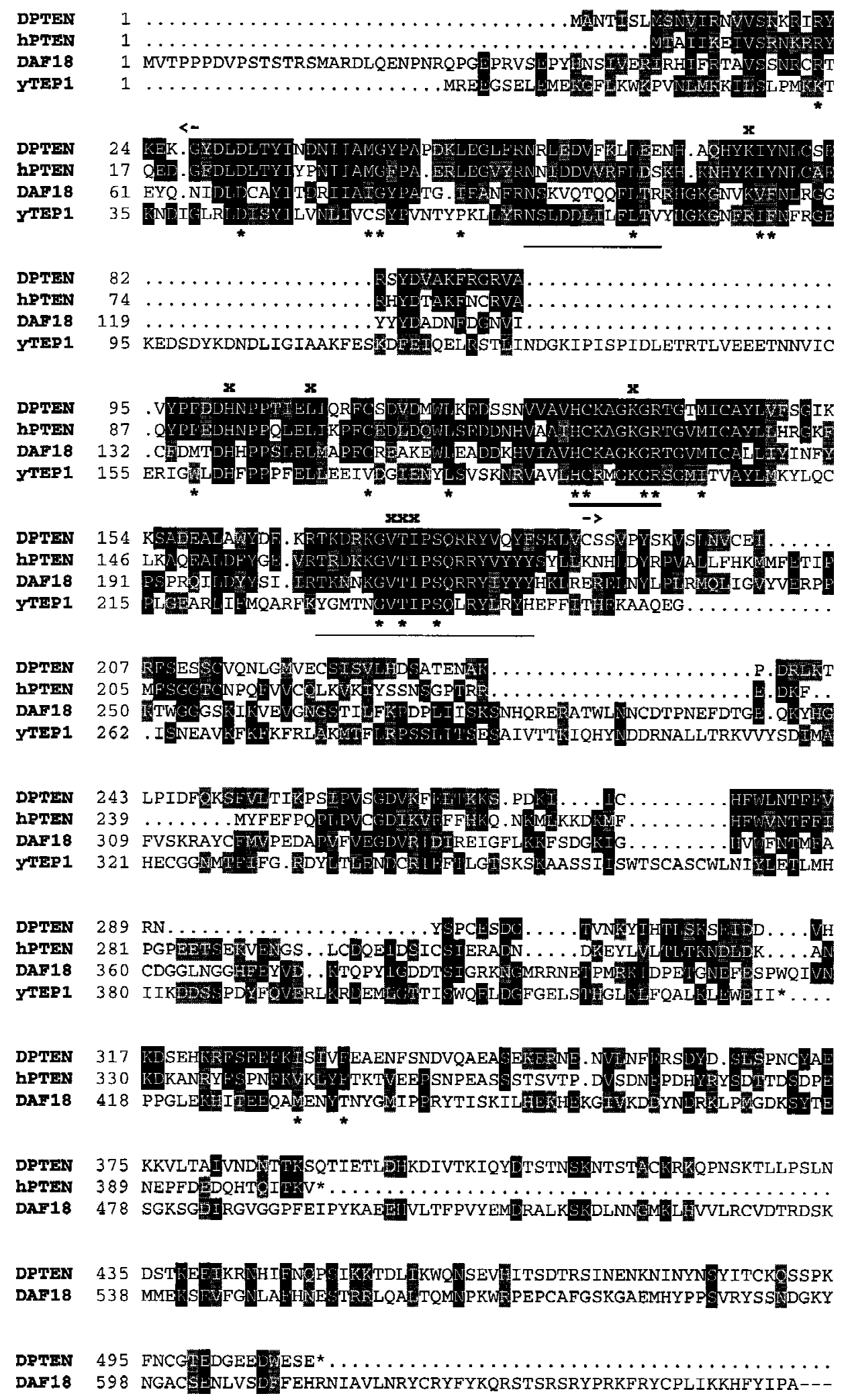

Figure 2. The PTEN protein family. The predicted DPTEN protein was compared with human PTEN (hPTEN; Steck et al. 1997; Li et al. 1997a), C. elegans PTEN (DAF-18; the carboxy-terminal portion of this 965-amino-acid protein is omitted; Ogg and Ruvkun 1998) and the yeast PTEN homolog TEP1 (yTEP1 or YNL128W; Li et al. 1997b). Identical amino acids are shaded in black and conservative changes in gray. The extent of the tensin domain is demarcated by arrows. Three regions within this domain that are required for phosphatase activity are underscored, including the highly conserved phosphatase signature motif (bold underline; Myers et al. 1997). The positions of missense mutations identified in cancer or in dominant genetic disorders are marked by asterisks (Maehama and Dixon 1999). All but one of the affected residues are identical in DPTEN. Crosses above the sequence highlight PTEN-specific amino acids that are not found in other known members of either the phosphatase or tensin families. 


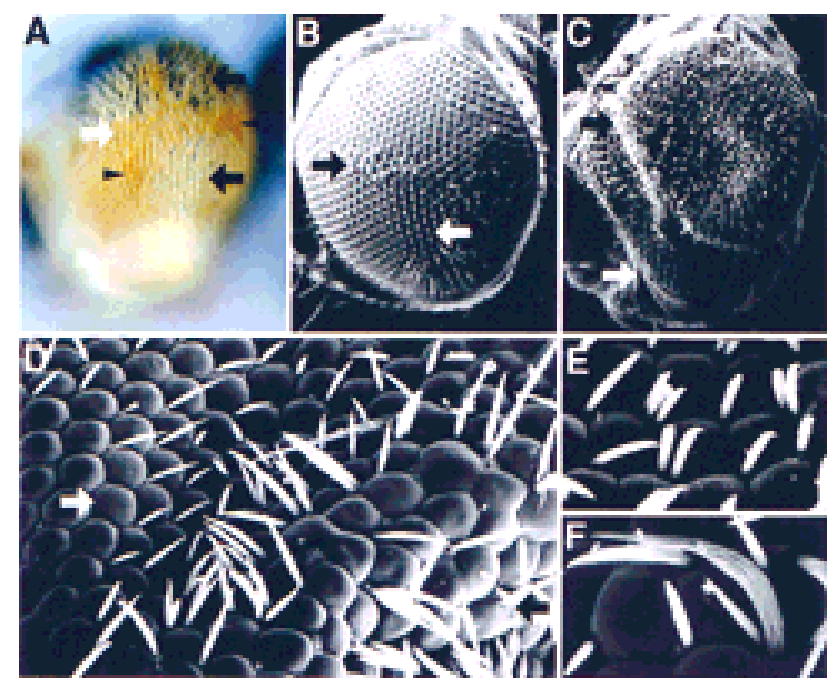

Figure 3. DPTEN regulates adult eye development. Light micrograph $(A)$ and scanning electron micrographs $(B-F)$ of eye clones homozygous for DPTEN ${ }^{1}(A, C-F)$ and $D f(2 L) 170 B(B)$. When compared with heterozygous tissue (white arrows in $A-D$ ), mutant regions (black arrows; in $A$, the mutant region is the nonpigmented region that does not express the $w^{+}$gene) contain enlarged ommatidial facets and aberrant clusters of up to four interommatidial bristles (seen at higher magnification in E). There are also more ommatidia in mutant clones than in adjacent wild-type twin spots (darker pigmented regions that express two copies of the $W^{+}$gene; marked with black arrowheads in $A$ ). Bristles are nonuniform in thickness, morphology, and length $(D-F$; shorter in $E$, several times longer in $F)$. The arrangement of actin bundles within these bristles is abnormal $(F)$. The genotypes of flies were $y, w, h s F L P 1 ; D P T E N^{1}, P\left[r y^{+}\right.$; hs-neo; FRT]40A/P[ry ; $\left.\mathrm{w}^{+}\right] 30 \mathrm{C}, \mathrm{P}\left[\mathrm{ry}^{+}\right.$; hs-neo; FRT]40A (A,CF) and $y, w, h s F L P 1 ; D f(2 L) 170 B, P\left[\mathrm{ry}^{+}\right.$; hs-neo; FRT]40A/P $\left[\mathrm{ry}^{+}\right.$; $\left.\mathrm{w}^{+}\right] 30 \mathrm{C}, \mathrm{P}\left[\mathrm{ry}^{+}\right.$; hs-neo; FRT $] 40 \mathrm{~A}(\mathrm{~B})$.

is mild compared with the effects of several other tumor suppressor mutations in Drosophila (Gateff and Mechler 1989; Xu et al. 1995).

Sections demonstrated that the number and identities of ommatidial cells were not obviously altered in DPTEN mutant clones, indicating that this gene does not have a major role in specifying cell fate in the eye. Analysis of nonpigmented mutant clones, however, revealed that the cell bodies of all mutant photoreceptors, but not their wild-type/heterozygous neighbors were greatly enlarged (Fig. 4A,C). By using a $w^{+}$-marked mutant chromosome to visualize the pigment cell layer in clones, it was possible to show that the volume of this layer was also increased (Fig. 4B). These cellular growth defects account for the increased size of mutant ommatidia. Furthermore, in mutant photoreceptors, the lightsensing rhabdomeres were elliptical and not circular in cross-section (Fig. 4A-D). This phenotype is observed in several mutants that are believed to affect assembly of the actin cytoskeleton (Fischer-Vize and Mosley 1994; Treisman et al. 1997; Thomas et al. 1998). Analysis of ommatidia containing both wild-type and mutant pho-

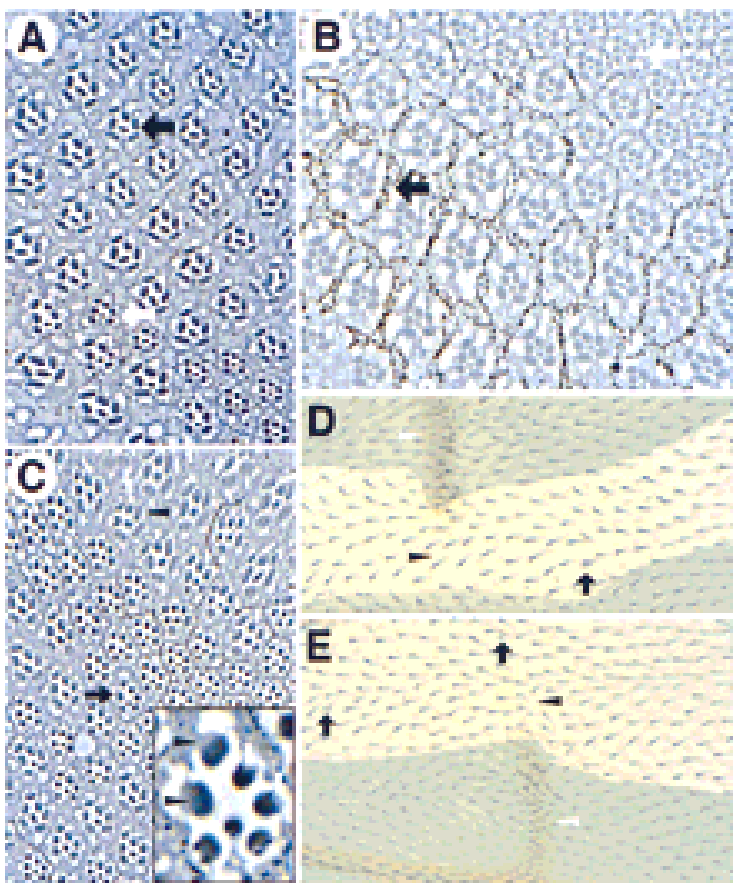

Figure 4. DPTEN controls cell size and architecture. $(A-C)$ Cross sections through eyes containing clonal tissue homozygous for a $w$ DPTEN ${ }^{1}$ chromosome $(A, C)$ and a $w^{+} D$ PTEN ${ }^{3}$ chromosome $(B)$. In $A$ and $C, W$ mutant photoreceptors can be distinguished from their phenotypically wild-type pigmented neighbors (cf. Fig. 3A). In contrast, $w^{+}$mutant pigment cells in $B$ carry two copies of the the $w^{+}$gene and are therefore more pigmented than their phenotypically wild-type neighbors. In $A$ and $B$, mutant (black arrow) and normal heterozygous (white arrow) tissues are indicated. Although the polarity of mutant ommatidia is largely unaffected, $w$ photoreceptor cell bodies $(A$; only low levels of pigment are seen in the heterozygous tissue in this sample) and $\mathrm{W}^{+}$pigment cells $(B)$ lacking DPTEN are increased in size. Also note the elliptical structure of mutant rhabdomeres. Rhabdomeres from mutant and phenotypically wild-type photoreceptors are marked with black and white arrowheads respectively in $C$. Multiple ommatidia of mixed genotype in $C$ reveal that the effects on size and rhabdomere morphology are cell-autonomous; the inset shows a single ommatidium (black arrow) in which the two mutant photoreceptors present are marked (black arrowheads) together with two phenotypically wild-type photoreceptors (white arrowheads). $(D, E)$ Wings containing clonal tissue homozygous for $D f(2 L) 170 B(D$; note that this fly also carried the DINK genomic rescue construct pWZ; Riesgo-Escovar et al. 1996) and for DPTEN ${ }^{1}(E)$. Clones were marked with the forked mutation (unshaded regions). Mutant cells are enlarged, and frequently cross-veins fail to extend from wild-type tissue (white arrowheads) into the mutant region (black arrowheads), although occasionally additional cross-veins are observed (data not shown). A minority of mutant cells contains duplicated wing hairs (black arrows). The genotypes of the flies were $y, w, h s F L P 1$; DPTEN ${ }^{1}, P\left[r y^{+}\right.$; hsneo; FRT $] 40 A / P\left[\mathrm{ry}^{+} ; \mathrm{w}^{+}\right] 30 \mathrm{C}, P\left[\mathrm{ry}^{+}\right.$; hs-neo; FRT $] 40 \mathrm{~A}(\mathrm{~A}, \mathrm{C}), \mathrm{y}$, w, hsFLP1; P[ry ; $\left.\mathrm{w}^{+}\right] 30 \mathrm{C}, \mathrm{DPTEN}{ }^{3}, \mathrm{P}\left[\mathrm{ry}^{+} ;\right.$hs-neo; FRT]40A/ $P\left[\right.$ ry $^{+}$; hs-neo; FRT]40A (B), y, w, hsFLP1, f36a; Df(2L)170B, $P\left[\mathrm{ry}^{+}\right.$; hs-neo; FRT]40A/P [f $] 30 \mathrm{~B}, \mathrm{P}\left[\mathrm{ry}^{+}\right.$; hs-neo; FRT]40A; $p W Z /+(D)$ and $y, w, h s F L P 1, f^{36 a} ; D P T E N^{1}, P\left[r^{+}\right.$; hs-neo; FRT $] 40 A / P\left[f^{+}\right] 30 B, P\left[\right.$ ry $^{+}$; hs-neo; FRT $] 40 A(E)$. 
toreceptors revealed that both the cell size and rhabdomeric phenotypes were cell-autonomous (Fig. 4C, inset).

Cells in the wing, each of which normally possesses a single wing hair, were also significantly enlarged in mutant DPTEN clones (Fig. 4E,F). Wing hairs were occasionally duplicated, a defect observed under conditions where the cytoskeleton of wing epithelial cells is disrupted (Eaton et al. 1996). In addition, crossvein formation in the wing, a process involving specialized contacts between wing epithelial cells and the extracellular matrix (ECM; Fristrom et al. 1993), was abnormal in mutant regions. In $\sim 70 \%$ of cases, anterior cross-veins were absent or partially absent in mutant clones, whereas the posterior cross-vein was affected in $\sim 35 \%$ of cases (Fig. $4 \mathrm{D}, \mathrm{E})$. Occasionally, extra wing vein material was observed (data not shown). Longitudinal veins were affected only very rarely.

\section{DPTEN and Dp110 act antagonistically in regulating cell size and proliferation}

In mammals it has been proposed that PTEN regulates cell growth via its PIP3 3-phosphatase activity (Furnari et al. 1998; Maehama and Dixon 1998; Myers et al. 1998; Stambolic et al. 1998). DPTEN may also regulate growth similarly, as recombinant DPTEN protein derived from the cDNA used in this study behaves like its mammalian counterpart, exhibiting both IP4- and PIP3 3-phosphatase activity (I. Pass, G. McConnachie, and C.P. Downes, pers. comm.).

Our analysis of mutant DPTEN clones in the eye and wing indicated that DPTEN not only regulates cell number, but also cell size, and this leads to hyperplastic growth in mutant tissue. Interestingly, both overexpression of a dominant negative form of Dp110/PI3-kinase (Leevers et al. 1996) and mutations either in the Drosophila insulin receptor (Chen et al. 1996) or in chico/
IRS1-4 (Böhni et al. 1999) reduce cell size as well as proliferation. Furthermore, overexpression of wild-type and activated forms of Dp110 produces similar size and proliferation defects to those seen in DPTEN mutant cells (Leevers et al. 1996). These observations are consistent with a model, in which growth is regulated in Drosophila by specific phosphoinositides, whose levels are controlled by the balance of DPTEN and Dp110 activities. To test this idea, we investigated whether growth could be affected by increasing DPTEN expression and whether DPTEN mutations interacted genetically with components of the insulin receptor/Dp110 signaling pathway.

A wild-type DPTEN cDNA was overexpressed in a specific region of the wing using the GAL4-UAS system (Brand and Perrimon 1993). Initially we used flies carrying a $d p p-G A L 4$ construct (Staehling-Hampton and Hoffmann 1994), which drives gene expression in cells that will normally populate the region between the third and fourth longitudinal wing veins (LIII and LIV). Overexpression of DPTEN reduced the size of this region by nearly $25 \%$ compared with wild type (Fig. $5 \mathrm{C}$ ). This was not a consequence of a general reduction in wing size in overexpressing flies, as an adjacent area of the wing between LIV and LV was essentially unaffected. The effect on wing area was similar to that produced by overexpression of $D p 110^{D 954 A}$, a dominant-negative, kinase-dead version of Dp110 (Fig. 5B; Leevers et al. 1996). The reduction was caused by both a decrease in cell size and cell number and was opposite to the effect of overexpressing an activated, membrane-associated form of Dp110, Dp110-CAAX, in the same region (Table 1; Leevers et al. 1996).

To test whether DPTEN's growth regulatory functions are primarily mediated by its effects on the insulin receptor-Dp110 signaling pathway and not by an independent signaling cascade, we searched for genetic effects of
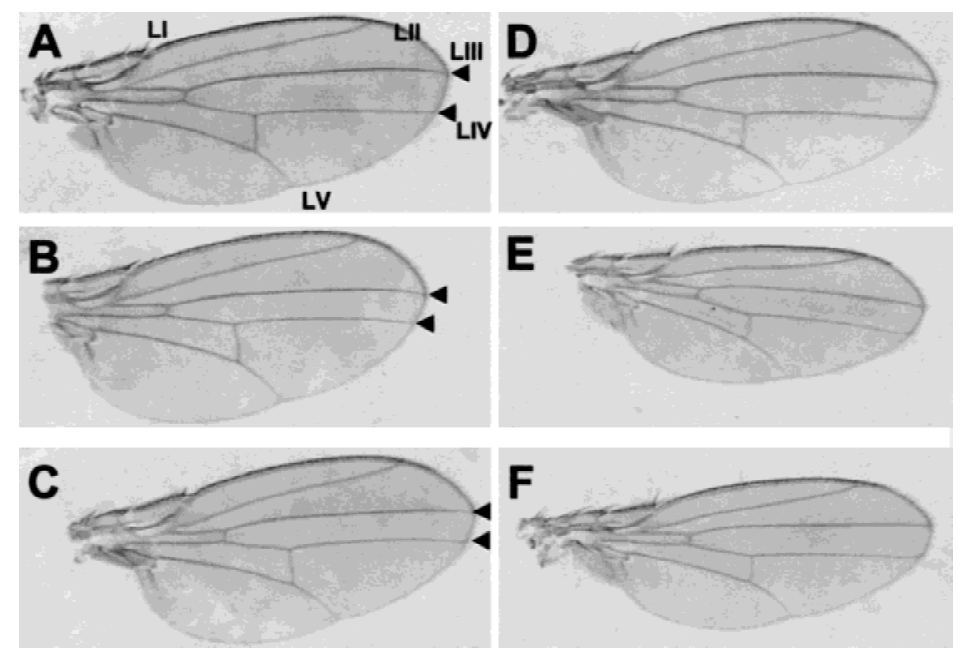
(A), w; $P\left[w^{+} ; U A S-D p 110^{D 954 A}\right] / P\left[w^{+} ; d p p-G A L 4\right](B), w ; P\left[w^{+} ; U A S-D P T E N(F F 20.2)\right] / S p ; P\left[w^{+} ; d p p-G A L 4\right] /+(C), w, P\left[w^{+} ; M S 1096-\right.$

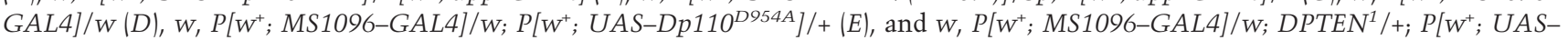
$\left.D p 110^{D 954 A}\right] /+(F)$.

Figure 5. DPTEN acts antagonistically to the PI3-kinase Dp110 in the wing. $(A-C)$ Wings from flies carrying the $d p p-G A L 4$ driver alone $(A)$, or expressing either a kinasedead, dominant-negative form of $D p 110, D p 110^{D 954 A}(B$; Leevers et al. 1996), or wild-type DPTEN (C) under the control of $d p p-G A L 4$ (Staehling-Hampton and Hoffmann 1994), which expresses GAL4 between wing veins LIII and LIV (arrowheads; wing veins LI to LV are marked in $A$ ). The area between these wing veins (called area I) is reduced by $\sim 25 \%$ in both $B$ and $C$ (see Table 1$)$. $(D-F)$ Wings from flies carrying the MS1096 driver alone $(D)$ or expressing $D p 110^{D 954 A}$ under the control of the MS1096 driver $(E, F)$, which expresses GAL4 in the wing, particularly on the dorsal surface (Leevers et al. 1996; Capdevila and Guerrero 1994), thereby reducing wing area in $E$ by $>30 \%$. In $F$, the fly is also heterozygous for DPTEN ${ }^{1}$, partially suppressing this phenotype. The wing area increases by $>8 \%$ compared with $E$, a $25 \%$ suppression of the reduced wing phenotype (see Materials and Methods). The genotypes of flies were $w ; . S p /+, P\left[w^{+}, d p p-G A L 4\right] /+$ 
Table 1. Overexpression of DPTEN reduces cell size and number in the adult wing

\begin{tabular}{|c|c|c|c|c|c|c|c|c|}
\hline 1 & 2 & 3 & 4 & 5 & 6 & 7 & 8 & 9 \\
\hline Genotype $^{a}$ & No. & $\begin{array}{c}\text { Area I } \\
\left(10^{5} \mu \mathrm{m}^{2}\right)^{\mathrm{b}}\end{array}$ & $\begin{array}{c}\text { Area II } \\
\left(10^{5} \mu \mathrm{m}^{2}\right)^{\mathrm{c}}\end{array}$ & $\begin{array}{l}\text { Area I/ } \\
{\text { area } \mathrm{II}^{\mathrm{d}}}^{\mathrm{a}}\end{array}$ & $\begin{array}{c}\text { Percent } \\
\text { difference } \\
\text { in area } \\
\text { ratio vs. } \\
\text { control }^{\mathrm{e}}\end{array}$ & $\begin{array}{c}\text { Cell density } \\
\text { of area I } \\
\left(10^{-3} \text { cells } / \mu \mathrm{m}^{2}\right)^{\mathrm{f}}\end{array}$ & $\begin{array}{l}\text { No. of } \\
\text { cells in } \\
\text { area } I^{\mathrm{g}}\end{array}$ & $\begin{array}{l}\text { Cell area } \\
\text { in area I } \\
\left(\mu \mathrm{m}^{2}\right)^{\mathrm{h}}\end{array}$ \\
\hline \multicolumn{9}{|l|}{ UAS Dp110 CAAX/Sp; TM6/ } \\
\hline $\begin{array}{l}\text { UAS Dp110 } 954 A / d p p-G A L 4 \\
\text { UAS DPTEN (FF20.2)/Sp; }\end{array}$ & 4 & $1.98 \pm 0.07$ & $3.00 \pm 0.06$ & $0.67 \pm 0.02$ & $-26 \pm 2$ & $6.5 \pm 0.2$ & $1290 \pm 30$ & $154 \pm 5$ \\
\hline $\begin{array}{l}\text { dpp-GAL4/+ }{ }^{i} \\
\text { UAS DPTEN }(F F 20.6) / S p\end{array}$ & 6 & $2.15 \pm 0.09$ & $2.93 \pm 0.10$ & $0.72 \pm 0.02$ & $-23 \pm 2$ & $6.0 \pm 0.1$ & $1340 \pm 10$ & $166 \pm 3$ \\
\hline$d p p-G A L 4 /+^{i}$ & 6 & $2.23 \pm 0.08$ & $3.03 \pm 0.07$ & $0.74 \pm 0.02$ & $-21 \pm 2$ & $6.0 \pm 0.1$ & $1280 \pm 50$ & $167 \pm 3$ \\
\hline $\begin{array}{l}\text { UAS WT Dp110/Sp; dpp-GAL4/+ } \\
\text { UAS Dp110 CAAX/Sp; }\end{array}$ & 5 & $2.88 \pm 0.05$ & $3.07 \pm 0.04$ & $0.94 \pm 0.01$ & $0 \pm 1$ & $5.4 \pm 0.1$ & $1510 \pm 50$ & $185 \pm 4$ \\
\hline$d p p-G A L 4 /+$ & 4 & $3.42 \pm 0.08$ & $2.99 \pm 0.10$ & $1.14 \pm 0.02$ & $+21 \pm 2$ & $5.1 \pm 0.2$ & $1740 \pm 30$ & $197 \pm 6$ \\
\hline
\end{tabular}

a Second chromosomes carrying UAS transgenes were transheterozygous with a chromosome carrying Sternopleural (Sp), which does not affect wing size significantly.

${ }^{\mathrm{b}} d p p-G A L 4$ primarily drives UAS-transgene expression in area I (the distal wing sector bounded by LIII, LIV, the wing margins, and the anterior cross-vein).

'In area II (the distal wing sector bordered by LIV, LV, the wing margins, and the posterior cross-vein), which lies in the posterior wing compartment, there is no $d p p-G A L 4$-driven UAS-transgene expression.

${ }^{\mathrm{d}}$ Area I was compared to area II to control for variation in animal and wing size in different UAS-transgene lines and flies.

eGenerated by comparing the value in column 5 with the control value in column 5.

${ }^{\mathrm{f}}$ Determined by counting number of wing hairs (each representing a single cell) in a defined rectangular area of $19,500 \mu \mathrm{m}^{2}$ adjacent to the intersection between the posterior cross-vein and LIV, and with the long edge of the rectangle parallel to LIII.

${ }^{\mathrm{g}}$ Generated by multiplying the values in column 7 by those in column 3 .

${ }^{\text {h}}$ Reciprocal of column 7 .

${ }^{i}$ Data for two independent UAS-DPTEN insertions (FF20.2 and FF20.6) are presented.

DPTEN alleles on mutant phenotypes associated with chico and Dp110. Because the IRS1-4 homolog Chico is believed to transmit only part of the growth-promoting signal from the Drosophila insulin receptor (Böhni et al. 1999), it is expected that Dp110 activity and consequently PIP3 production should be reduced, but not eliminated in chico mutants. If DPTEN acts downstream of Chico to antagonize Dp110 by converting most newly synthesized PIP3 into PIP2, loss of DPTEN should produce excess levels of PIP3, even in a chico mutant.

In eye clones homozygous for specific chico alleles or for the deficiencies, $D f(2 L) 147 F$ and $D f(2 L) 41 C$, that eliminate chico, bsk, and in the latter case Dror activities (Fig. 1A), cells were reduced significantly in size and number relative to the twin spot (Böhni et al. 1999; data not shown). In fact, most twin spots generated by recombination are not associated with a visible mutant clone (see Böhni et al. 1999 for discussion). In contrast, as described above, cells homozygous for $D f(2 L) 170 B$, a deficiency that is null for chico, DINK, Dror, and DPTEN function were nearly as enlarged as cells mutant for specific DPTEN alleles (Figs. 3B and 4E). The external overgrowth phenotype observed in these clones (e.g., see Fig. $3 \mathrm{~B})$ was not seen in flies also carrying the DPTEN genomic construct, viv1 (Fig. 1A), demonstrating that loss of DPTEN function has an essential part in generating this phenotype. Multiple lines of evidence show that the two other genes deleted in this deficiency, DINK and Dror, do not have a significant role in suppressing the chico phe- notype. First, as discussed above, removal of both these genes in $D f(2 L) 41 C$ clones within the eye did not affect the chico phenotype; the clones were small and frequently not visible externally. Furthermore, DINK has no reported role in cell growth control and Dror is a neural-specific gene (Wilson et al. 1993), that would not be anticipated to have a general effect on growth in structures like the wing. Finally, neither a $D J N K$ genomic construct (i.e., see Fig. 4D) nor a Dror genomic construct (data not shown) had any effect on the external growth defects produced by the $D f(2 L) 170 B$ deficiency. These results demonstrate that DPTEN is epistatic to chico, strongly supporting a role for DPTEN in modulating the Chico-dependent growth promoting signal.

If DPTEN acts in opposition to Dp110 to control growth, coexpressing DPTEN with Dp110 should suppress the growth-promoting effects of the latter molecule. When the wild-type $D p 110$ gene was expressed throughout the posterior compartment of the wing using an engrailed (en)-GAL4 driver (a gift from A. Brand, Wellcome/CRC Institute, Cambridge, UK), almost all animals died as larvae or pupae, and those animals that survived had distorted and crumpled wings. The posterior compartment in these wings was greatly enlarged relative to the anterior compartment (Fig. 6B). Overexpression of DPTEN using the same driver also decreased viability, but in the $20 \%$ of flies that eclosed from pupae, the posterior compartment was significantly reduced in size (Fig. 6C). Flies overexpressing both genes had a 

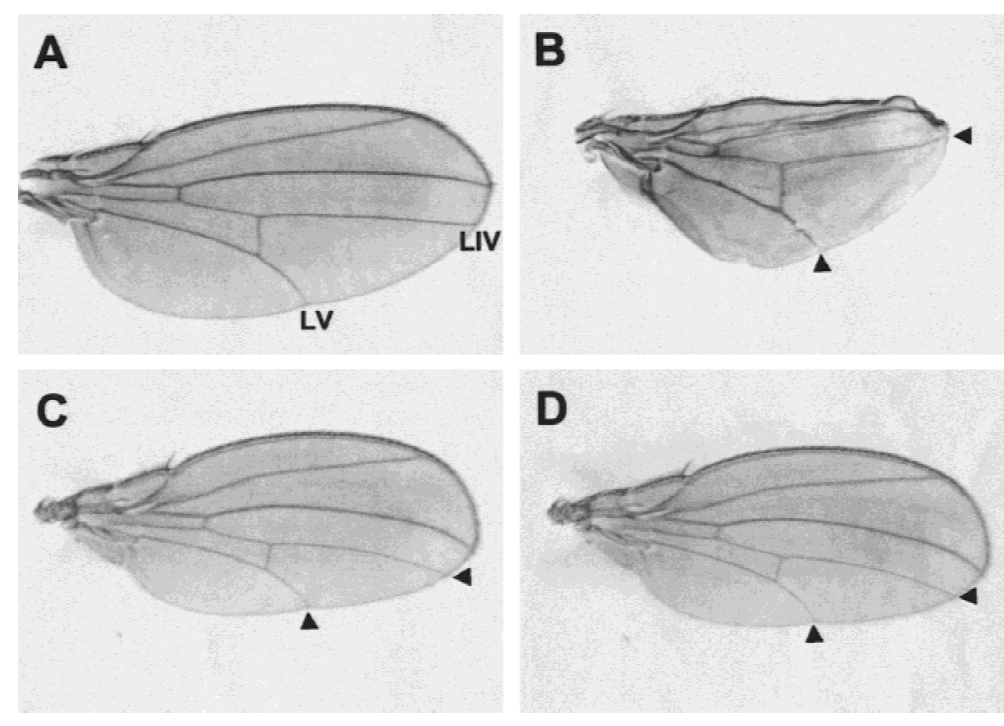

Figure 6. DPTEN completely suppresses the growthpromoting activity of overexpressed $D p 110$ in the wing. Wings from flies carrying the en-GAL4 driver alone $(A)$, or overexpressing $D p 110(B), D P T E N(C)$, or both Dp110 and DPTEN (D) under en-GAL4/UAS control in the posterior compartment of the wing. The compartment boundary lies just anterior to LIV; the posterior compartment therefore includes wing veins LIV and LV (labeled in $A$ and marked with arrowheads in the other panels). Although the wing produced by overexpression of Dp110 is crumpled and distorted, note that its posterior compartment is enlarged compared with the anterior compartment, a phenotype most clearly seen in the region between LIV and LV. In contrast, this area is reduced in flies overexpressing DPTEN and overexpressing the combination of DPTEN and Dp110. The genotypes of flies were $W ; P\left[W^{+} ;\right.$en-GAL4] $/+(A), w ; P\left[W^{+} ; U A S_{-}\right.$ $D p 110] / P\left[w^{+}\right.$; en-GAL4] (B), w; $P\left[w^{+} ; U A S-D P T E N\right] /$ $P\left[w^{+}\right.$; en-GAL4] $(C)$, and $W ; P\left[w^{+} ; U A S-D p 110\right], \mathrm{P}\left[\mathrm{w}^{+}\right.$; UAS-DPTEN $] / \mathrm{P}\left[\mathrm{w}^{+}\right.$; en-GAL4] $(D)$. much higher viability than those expressing either gene alone ( $>95 \%$ of pupae eclosed). Furthermore, the posterior compartment of the wing was nearly as reduced in area as in animals overexpressing only DPTEN (Fig. 6D; Materials and Methods), showing that DPTEN completely suppresses the growth-promoting effects of Dp110 in this system.

Finally, we also observed that DPTEN mutations dominantly modify Dp110-induced phenotypes in several different allelic combinations. For example, overexpression of a kinase-dead form of Dp110 under the control of the wing-specific GAL4 driver MS1096 leads to a $30 \%$ reduction in wing area (Leevers et al. 1996). This was partially suppressed in flies heterozygous for $D P T E N^{1}$ or $D f(2 L) 170 B$ (Fig. 5D-F).

Taken together, these data demonstrate that DPTEN controls growth in vivo by antagonizing Chico/Dp110 signaling, and are consistent with a model in which DPTEN primarily acts by dephosphorylating the growth regulator PIP3. We conclude that the ratio of DPTEN and PI3-kinase activities during development is a key regulatory determinant of growth in flies. Our results provide important in vivo support for the hypothesis that a change in this ratio in PTEN-linked cancers and dominant genetic disorders underlies the growth defects in these diseases.

\section{DPTEN affects the organization of the actin cytoskeleton in differentiating adult cells}

The bristle, hair, and rhabdomere phenotypes observed in DPTEN mutant tissue have not been reported in flies defective in insulin or Dp110 signaling, indicating that unlike the DPTEN-linked growth defects, these effects are probably not caused simply by an increase in PIP3 levels. We analyzed DPTEN mutant clones in the larval imaginal disks to determine whether there was an underlying defect in microfilament organization during differentiation that might explain these phenotypes.
Consistent with the adult eye phenotype, DPTEN mutant ommatidial preclusters in the eye imaginal disk were more widely spaced (Fig. 7), and specific staining of photoreceptors indicated that these cells were enlarged in mutant clones (data not shown). Phalloidin staining demonstrated that there were defects in the actin cytoskeletal network in mutant tissue (Fig. 7B,D). In particular, analysis of mutant photoreceptors revealed disorganization in the most basal part of the apical cytoskeleton, the scaffold on which apical photoreceptor projections are normally assembled in the disk. An altered distribution of neighboring actin microfilaments was also observed (Fig. 7B,D). Because rhabdomere assembly in photoreceptors requires the apical cytoskeleton (Wolff and Ready 1993), this defect may account for the rhabdomeric phenotype seen in adult DPTEN mutant ommatidia. Analysis of the wing imaginal disk also showed disturbed cytoskeletal organization in mutant tissue (data not shown). Therefore, although DPTEN mutant cells can still assemble actin microfilaments, the subcellular regulation of this process appears to be abnormal, potentially accounting for the structural defects in adult bristles, rhabdomeres, and wing hairs.

\section{Discussion}

The human tumor suppressor gene PTEN has been implicated in a wide range of cancers (Myers and Tonks 1997; Smith and Ashworth 1998), but the precise mechanisms by which it inhibits tumorigenic growth and its developmental roles in vivo are not established. Here we describe the cloning and genetic characterization of the Drosophila PTEN homolog, DPTEN, and demonstrate that although this gene has remarkably little influence on cell-type specification, it has a central role in tissue growth, reducing both cell size and number. Genetic experiments indicate that DPTEN's effects on growth are primarily mediated by its antagonistic effect on Chico/ Dp110 signaling. We provide evidence that DPTEN also 


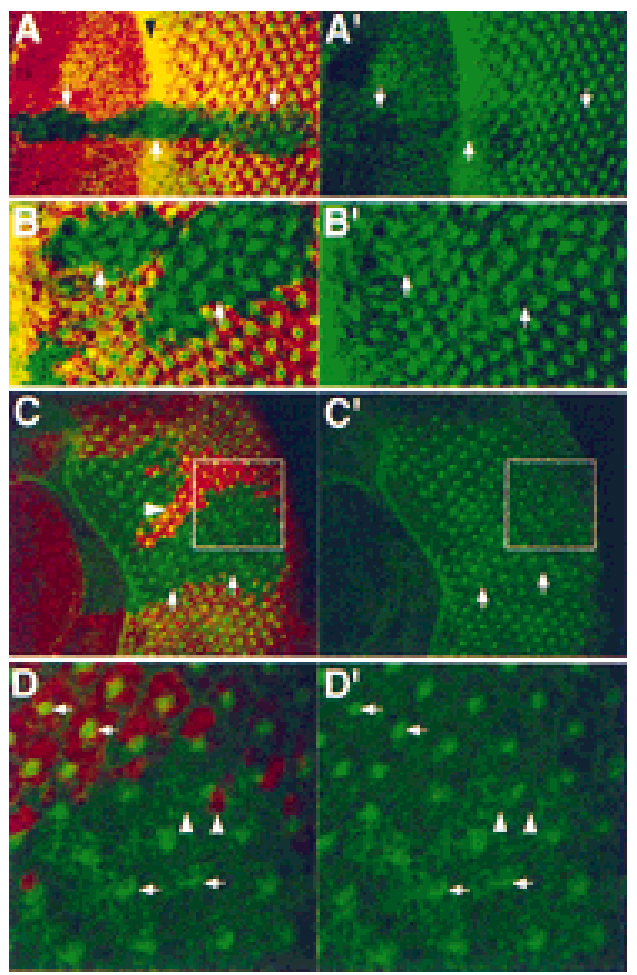

Figure 7. DPTEN affects subcellular localization of the actin cytoskeleton in the developing eye. Panels show confocal micrographs of third-instar larval eye imaginal disks containing $D P T E N^{1}$ clones. Anterior is left in all panels. The mutant area is marked by the absence of $\operatorname{lac} Z(A, B)$ or GFP $(C, D)$ expression (lacZ- and GFP-positive tissue is shown in red), whereas actin elements are visualized by the actin binding molecule phalloidin (coupled to FITC in $A$ and $B$ and to rhodamine in $C$ and $D$; green in all panels). The overlay is shown on the left side. White arrows in $(A-C)$ point to the mutant area, which does not stain red. (A) Eye disk with mutant clone containing both uncommitted cells, found anterior to (left half of panel) and within the morphogenetic furrow (black arrowhead), and posterior cells within developing ommatidial preclusters. Actin elements within developing ommatidial clusters are shown in more detail in $B$. Both $A$ and $B$ show stacked confocal sections, which scan a depth of $\sim 5 \mu \mathrm{m}$ in the apical half of the photoreceptor. Mutant cells have an altered distribution of actin microfilaments compared with their phenotypically wild-type neighbors, i.e., in the developing ommatidial clusters shown in $B$, there is a more intricate network of microfilaments in mutant cells. $C$ and $D$ show low and high magnification views, respectively, of a large DPTEN clone. Note in $C$, the reduced number of cells in the wild-type twin spot (bright red cells marked with white arrowhead) and the boxed area enlarged in $D . D$ shows the altered organization of the apical cytoskeleton and neighboring microfilaments compared with wild-type tissue (apical projections marked with white arrows). In some instances, wild-type and mutant photoreceptors within the same cluster (marked with white arrowheads) appear to display normal and mutant phenotypes, respectively.

regulates the actin cytoskeletal network via a currently undefined mechanism and suggest that this permits changes in cell size to be coordinated with cytoskeletal modifications at the cell surface that may affect properties such as cell spreading.

\section{DPTEN is a highly evolutionarily conserved lipid and protein phosphatase}

The predicted DPTEN protein shares considerable homology with its human counterpart. The similarity extends beyond the highly conserved amino-terminal tensin and phosphatase domains into the carboxy-terminal half of the molecule (Fig. 2), which is more divergent in the yeast and C. elegans PTEN homologs. This carboxyterminal region currently has no defined function, but it is affected by a number of missense mutations that are linked to cancer and dominant genetic disease in humans (for review, see Maehama and Dixon 1999). Clearly the region could have a regulatory role or localize the PTEN molecule to the appropriate cellular compartment. In this context, however, it is important to note that the carboxyl terminus of human PTEN, which shares similarity to known binding sites for PDZ domains (Teng et al. 1997), is not conserved in DPTEN.

\section{DPTEN regulates cell size and number in development}

We identified several noncomplementing mutations that by deficiency mapping are located within or close to the DPTEN gene. Two of these mutations, which we have used in this study, behave similarly to a deficiency for DPTEN and are therefore likely to be strong alleles. In combination with a DPTEN deficiency, these mutations cause lethality during late embryonic and early first-instar larval development. This lethality is rescued by a single copy of the DPTEN genomic locus and by lowlevel expression of a heat shock-DPTEN cDNA construct at noninducing temperatures, demonstrating that the complementation group is allelic to DPTEN.

Because DPTEN mutant animals die with no obvious phenotypes, we studied the function of this gene by inducing mutant clones in developing larvae. Analysis in both the adult eye and wing demonstrated that DPTEN reduces cell size, a result supported by experiments where the wild-type gene was overexpressed in the wing. The effect of DPTEN is most conspicuous in clones within the eye, where a severalfold increase in mutant photoreceptor size distorts the highly ordered ommatidial array. Growth is affected in multiple cell types in the eye, including pigment cells and all photoreceptors, indicating that DPTEN is a general regulator of cell size.

Within the eye, the number of cells in homozygous DPTEN mutant clones was consistently greater than the number of cells in the associated wild-type twin spot, indicating that DPTEN also affects tissue growth by regulating cell number. This conclusion is supported by our analysis of the effects of DPTEN overexpression in the wing, where both cell size and number are reduced. Because DPTEN mutant clones also appear to contain more cells than their corresponding twin spots in larval 
eye imaginal disks, these effects on cell growth are probably at least partly caused by changes in proliferation rate, a conclusion that was also made in an analysis of chico mutants (Böhni et al. 1999).

\section{DPTEN regulates tissue growth and body size} by antagonizing the Chico/Dp110 signaling pathway

In mammalian cell culture, PTEN has been shown to regulate cell proliferation and survival via its lipid phosphatase activity, which converts PIP3 into PIP2 (Furnari et al. 1998; Stambolic et al. 1998). A series of genetic experiments presented here has demonstrated that in living flies, DPTEN affects both cell size and number by antagonizing the effects of Dp110/PI3-kinase, the enzyme that converts PIP2 into PIP3.

We have shown that phenotypes in homozygous DPTEN mutant cells are very similar to the growth phenotypes seen in cells overexpressing a constitutively activated form of Dp110 (Leevers et al. 1996). In addition, the effects of overexpressing a dominant-negative form of Dp110 in the wing (Fig. 5B) can be phenocopied by overexpressing wild-type DPTEN (Fig. 5C). DPTEN mutations show a significant dominant suppression of wing growth phenotypes induced by overexpression of dominant-negative Dp110. Furthermore, overexpression of DPTEN (under an en-GAL4 driver) completely suppresses the growth-promoting activity of overexpressed Dp110. The importance of DPTEN in negatively regulating the growth-promoting effects of insulin signaling in vivo, however, is best illustrated in homozygous clones mutant for both chico and DPTEN. In these clones, the reduced growth phenotype normally seen in chico mutant cells is masked completely by the overgrowth phenotype associated with loss of DPTEN function, suggesting that DPTEN normally has a critical role downstream of Chico in maintaining growth-promoting signals at nonhyperplastic levels.

Böhni et al. (1999) have recently shown that insulin receptor signaling controls tissue and body size in Drosophila. Our data extend this model and show that it is the balance of DPTEN and Dp110 activities in this pathway that acts as the key determinant of growth in the fly (Fig. 8). Although we cannot eliminate the possibility that protein substrates of these molecules have a role in this process, it seems likely that Dp110 and DPTEN primarily act by controlling the levels of the phosphoinositide growth regulator PIP3.

Our phenotypic analysis supports the idea that the Chico/Dp110/DPTEN-regulated growth control signal, which is stimulated by insulin receptor activation, behaves differently from several other signals that affect cell proliferation in flies. For example, overexpression in the wing of E2F, a positive regulator of cell proliferation, increases cell number, but does not appear to affect protein synthesis rates and therefore produces a concomitant reduction in cell size, so that wing area is unaffected (Neufeld et al. 1998). In contrast, an increased level of Dp110 activity relative to DPTEN produces increased cell numbers and growth, a phenotype that can be easily

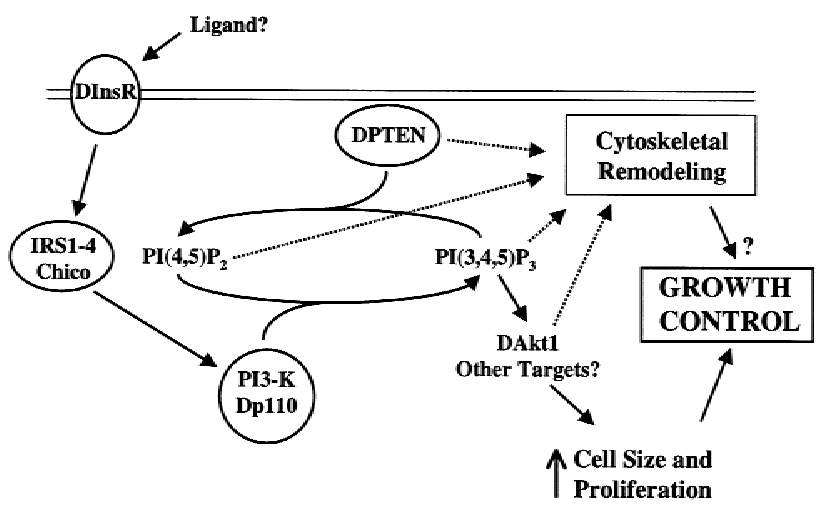

Figure 8. Schematic diagram illustrating a model for the antagonistic roles of Dp110 and DPTEN in Drosophila growth control. In mammals, Drosophila, and C. elegans, PI3-kinase activity is regulated by signaling cascades that include the insulin receptor and IRS1-4 (Chen et al. 1996; Maehama and Dixon 1998; Ogg and Ruvkun 1998; Böhni et al. 1999). Disruption of this pathway in mice (Baker et al. 1993; Liu et al. 1993) and Drosophila (Böhni et al.1999) has shown that insulin receptor signaling controls tissue and body size. Our data indicate that DPTEN antagonizes this pathway in flies and decreases cell size and number. It seems likely that DPTEN's effects are largely mediated by reducing PIP3 levels and the activity of its putative downstream targets, including Dakt1. DPTEN's protein phosphatase activity, however, could also have a role (see Gu et al. 1999). DPTEN also appears to regulate organization of the actin cytoskeleton via currently uncharacterized mechanisms (dotted lines) that may involve either its protein or lipid phosphatase activity. DPTEN's effects on the cytoskeleton may influence its tumor suppressing activity by modulating processes such as cell spreading and invasiveness.

scored in the highly organized structures of the Drosophila wing and eye. Analysis of mice lacking components of the insulin signaling cascade (e.g., Baker et al. 1993; Liu et al. 1993) suggests that this pathway also controls tissue growth in mammals. It remains unclear, however, whether cell size as well as proliferation is affected in these animals or in humans with genetic defects in PTEN.

\section{DPTEN affects actin cytoskeletal organization in multiple cell types}

Further analysis of DPTEN mutant cells in both the wing and eye led us to two further conclusions regarding the function of this gene in development. First, DPTEN generally does not have a role in cell fate specification. This contrasts with studies of PTEN mutant mouse embryos, where early patterning defects have been observed (Di Cristofano et al. 1998; Suzuki et al. 1998), although the cellular basis of these phenotypes has not been determined. Perhaps PTEN's phosphatase activity is required for specific cell-cell or cell-matrix communication events controlling patterning in these embryos. Likewise, the defects in cross-vein formation in DPTEN mutant clones in the fly wing might be explained in this way. Loss of DPTEN function invariably produces hyper- 
plastic and not neoplastic growth in flies. In contrast, Di Cristofano et al. (1998) have found that embryonic stem (ES) cells homozygous mutant for PTEN can form neoplastic tumors in chimaeric mice. These cells, however, may carry mutations in other genes that contribute to this phenotype.

A second notable feature of DPTEN mutant cells is that they show phenotypes consistent with defects in organization of the actin cytoskeleton. The bristle, rhabdomere, and wing hair phenotypes observed in adult clones indicate that the cytoskeleton is probably affected in many cell types and our analysis of the eye imaginal disk suggests that the defects arise early in the development of these cells. One interesting hypothesis that remains to be tested is that these effects on the cytoskeleton may have an important role in regulating cell growth by controlling spreading and shape change at the cell surface. It is intriguing to speculate that loss of DPTEN function might alter communications between the peripheral actin cytoskeleton, the plasma membrane and the extracellular matrix, and therefore affect the invasiveness of cells, as has been suggested from studies in mammalian cell culture (Tamura et al. 1998). To resolve this issue, we are currently investigating whether DPTEN can modulate cell migration or invasiveness in several cell types during development.

Interestingly, overexpression of a constitutively activated form of Dp110 in the developing eye or wing does not appear to produce rhabdomeric, bristle, or wing hair phenotypes similar to those seen in DPTEN mutant cells (Leevers et al. 1996; data not shown), suggesting that these DPTEN-associated mutant defects may not result from increased PIP3 levels. These observations, however, do not eliminate the possibility that DPTEN modulates cytoskeletal assembly via localized production of the known cytoskeletal regulator PIP2 (Toker 1998), a process that might be enhanced and not inhibited by increased Dp110 activity. Consistent with this idea, overexpression of dominant-negative Dp110, which should reduce PIP3 and thereby might suppress the production of localized PIP2 by DPTEN, does lead to defects in the apical cytoskeleton of pupal photoreceptors (Leevers et al. 1996). In contrast, overexpression studies in mammalian cell culture have implicated PTEN's protein phosphatase activity in the disassembly of the cytoskeleton at focal adhesions (Tamura et al. 1998). Further experiments in flies, including rescue of the cytoskeletal defects using DPTEN mutant proteins that specifically lack lipid phosphatase activity, should clarify which catalytic activity of DPTEN is important in these developmental functions.

\section{Drosophila as a model system to study PTEN function}

In this report, we have provided in vivo evidence that PTEN acts as a general growth regulator by antagonizing the Chico/Dp110 signaling pathway. Taken together with the results of Böhni et al. (1999), our data suggest that the balance of Dp110 and DPTEN activities in flies has a remarkably specific role in the regulation of cell size and number. Future experiments in this powerful genetic system should lead to the identification of regulators and downstream molecules in this pathway, which may ultimately be suitable as novel targets for tumor therapy.

\section{Materials and methods}

Drosophila strains and culture

All fly strains were maintained at $18^{\circ} \mathrm{C}$ or $25^{\circ} \mathrm{C}$ on standard cornmeal agar medium (Cline 1978), supplemented with dried live yeast. Genetic symbols are as described in Lindsley and Zimm (1992). Balanced flies carrying $D f(2 L) 170 B, D f(2 L) 41 C$, and $D f(2 L) 147 F$ chromosomes (Sluss et al. 1996) were kindly provided by Kate Beckingham, Houston, Texas.

\section{Generation of DPTEN alleles}

Mutant alleles were generated with ethylmethyl sulphonate using standard procedures (Ashburner 1989). DPTEN ${ }^{1}$ was induced on a wild-type Canton S chromosome and subsequently recombined on to a $P[F R T] 40 A$ chromosome (Xu and Rubin 1993), whereas $D P T E N^{3}$ was induced on a $P\left[W^{+}\right] 30 C P[F R T] 40 A$ chromosome (for detailed genotypes, see Figs. 3 and 4 ).

\section{Molecular characterization of DPTEN}

DPTEN-containing genomic clones were isolated from a Drosophila genomic library (constructed by R. Davis, Baylor College of Medicine, Houston, TX) in $\lambda$ DASH II (Stratagene) using a Dror cDNA probe (Fig. 1A; Wilson et al. 1993). DPTEN cDNAs were subsequently isolated from a third-instar larval brain cDNA library (Wilson et al. 1993). Several independent DPTEN genomic and cDNA clones were sequenced on both strands. Oligonucleotide primers were synthesized by Oswel DNA Service (UK) and MWG-Biotech UK Ltd. DNA sequence analysis was carried out by the Advanced Biotechnology Centre at Charing Cross and Westminster Medical School. Sequence data were assembled using the GCG software package (University of Wisconsin, Madison).

A putative, full-length cDNA (clone 5C) including the entire DPTEN open reading frame (ORF) (Fig. 1B) was primarily used in this study. This cDNA was $2.2 \mathrm{~kb}$ in length with $294 \mathrm{bp}$ upstream and 397 bp downstream of the major ORF. Another prematurely terminated cDNA (clone 6C) and a truncated DPTEN cDNA from the EST database (GM01261) extended in the 5' direction from the $3^{\prime}$ upstream translated region (UTR) to positions 1396 and 1655 in the genomic sequence, respectively (Fig. 1B). Sequence analysis revealed that the former of these two transcripts was alternatively spliced, removing exon 11 and resulting in a short carboxy-terminal extension to the conceptual DPTEN protein (Fig. 1B). Compared with the DPTEN genomic sequence (Fig. 1B), cDNA clones showed the following polymorphisms: position 91, $\mathrm{A} \rightarrow \mathrm{C}$ (clone $5 \mathrm{C}$ ); 907, $\mathrm{C} \rightarrow \mathrm{A}$ $(5 \mathrm{C}) ; 1159, \mathrm{C} \rightarrow \mathrm{A}(5 \mathrm{C}) ; 1645, \mathrm{~T} \rightarrow \mathrm{C}(5 \mathrm{C}) ; 3001-3009$, deletion $(5 \mathrm{C}, 6 \mathrm{C}$, not GM01261); 3504, C $\rightarrow \mathrm{A}(5 \mathrm{C}, 6 \mathrm{C}) ; 3557, \mathrm{C} \rightarrow \mathrm{T}$ (GM01261); 3603, T $\rightarrow$ G (5C). Some cDNAs also have an extended 3' UTR (to 4203 instead of 4197; 5C, 6C). Only the polymorphic deletion alters the encoded DPTEN protein. The sequences of the DPTEN genomic locus, cDNA clone 5C and cDNA clone 6C have been deposited in the GenBank data library under accession numbers AF201904, AF201905 and AF201906, respectively.

Alignments were generated by the PILEUP program, and con- 
served amino acids were highlighted using the Boxshade program (www.isrec.isb-sib.ch/software/BOXform.html), except $\mathrm{A} \rightarrow \mathrm{S}$ and $\mathrm{W} \rightarrow \mathrm{Y}$ were not considered as conservative changes.

\section{Germ-line transformation and rescue experiments}

The genomic rescue construct vivI was generated by subcloning a 6.3-kb genomic NotI-SalI fragment (Fig. 1A) into the transformation vector pW8 (Ashburner 1989). The genomic NotI site was derived from flanking $\lambda$ DASH II sequences. For the heat shock construct, the longest available DPTEN cDNA (clone $5 \mathrm{C}$ ), encoding the shorter form of DPTEN (see Results; Fig. 1B), was cloned into the NotI site of a modified hsp70 construct in pCaSpW18, a pCaSpeR derivative (Ashburner 1989). Qiagen DNA preparations of these constructs were microinjected into cleavage stage $w ; K p p P[\Delta 2-3] 99 B$ embryos. For both constructs, at least three independent lines rescued all potential combinations of DPTEN alleles to viability at $25^{\circ} \mathrm{C}$, with the genomic construct giving a higher proportion of rescued flies.

\section{Clonal analysis}

Homozygous DPTEN mutant clones were generated using the FLP/FRT system (Xu and Rubin 1993) and marked by the absence (or duplication) of a $\mathrm{W}^{+}$construct in the adult eye, forked ${ }^{+}$ in the wing and lacZ or GFP expression in larval imaginal disks. Recombination was induced by a 1 -hr heat shock at $37^{\circ} \mathrm{C}$ between 24 and $72 \mathrm{hr}$ after egg laying (AEL). Mutant clones induced by heat shocks between 24 and $48 \mathrm{hr}$ were large (often at least 50-100 ommatidia) and frequently contained at least three to four times as many ommatidia as the twin spot. Many of these clones extended beyond the boundary of the eye and were therefore not included in the analysis of ommatidial number discussed in the text. S.E.M. and sectioning of adult eyes were performed by standard procedures. Imaginal disks were stained according to Xu and Rubin (1993).

\section{Wing analysis}

Selected transgenes were overexpressed in the entire wing and in specific regions of the wing using the upstream activating sequence (UAS)-GAL4 system (Brand and Perrimon 1993). decapentaplegic (dpp)-GAL4 (Staehling-Hampton and Hoffmann 1994) was employed to drive expression between longitudinal wing veins III and IV (LIII and LIV). Adult wings were dehydrated in ethanol and mounted in DPX. Wing areas were measured using the Leica Q500MC image analysis package. For each genotype, at least four wings derived from different female flies were analyzed. Area measurements of two distal wing sectors were made: area I, delineated by LIII, LIV, the anterior crossvein and the wing margin; and area II, bounded by LIV, LV, the posterior cross-vein and the wing margin. The wing veins themselves were not included in these areas. Because the UAS-linked genes were expressed in area I, but not in area II, comparison of these areas controlled against differences in overall wing area in flies of different genotypes or within populations of the same genotype and reduced the variability in measurements. Wing area differences were assessed for significance by a standard two-tailed $t$-test $(P<0.001)$. In comparison with wild type, overexpression of wild-type Dp110 did not produce a significant increase in either area I or the area I/area II ratio.

Dp110 and DPTEN were overexpressed in the posterior compartment of the wing using flies carrying an en-GAL4 driver. Overexpression of either gene alone produced a significant level of larval/pupal lethality, which was almost entirely suppressed when the genes were coexpressed (a result obtained with two different UAS-DPTEN insertions). The size of the posterior compartment in wings overexpressing Dp110 was difficult to measure because the wings were crumpled and distorted. Mean wing areas were calculated using at least four females of the following genotypes: $W$; en-GAL4) $\left(8.51 \times 10^{5}\right.$ $\left.\mu^{2} \pm 0.31 \times 10^{5}\right), w ; U A S-D P T E N / e n-G A L 4\left(5.88 \times 10^{5} \mu \mathrm{m}^{2} \pm\right.$ $\left.0.21 \times 10^{5}\right), w ; U A S-D P T E N, U A S-D p 110 /$ en-GAL4 $\left(6.72 \times 10^{5}\right.$ $\mathrm{um}^{2} \pm 0.16 \times 10^{5}$.

$U A S-D p 110^{D 954 A}$ was overexpressed in the entire wing using MS1096-GAL4 (Capdevila and Guerrero 1994), which expresses GAL4 particularly strongly in the dorsal wing pouch and in this combination reduces wing area by $\sim 30 \%$. The effect of the $D P T E N^{1}$ and $D f(2 L) 170 B$ chromosomes on this phenotype was tested. Mean wing areas were measured for at least four female flies of the following genotypes: $W, M S 1096-G A L 4 / W$ $\left(1.77 \times 10^{6} \quad \mathrm{~mm}^{2} \pm 0.04 \times 10^{6}\right), \quad w_{,}$MS1096-GAL4/W; UAS$D p 110^{D 954 A} /+\left(1.18 \times 10^{6} \mathrm{um}^{2} \pm 0.02 \times 10^{6}\right), w, M S 1096-G A L 4 /$ W; DPTEN ${ }^{1} /+; \quad U A S-D p 110^{D 954 A} /+\left(1.28 \times 10^{6} \mu \mathrm{m}^{2} \pm 0.04 \times\right.$ $\left.10^{6}\right)$, and $W, M S 1096-G A L 4 / W ; D f(2 L) 170 B /+$; UAS-

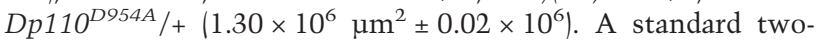
tailed $t$-test $(P<0.001)$ revealed that both DPTEN mutations suppressed the reduced wing phenotype significantly.

\section{Acknowledgments}

We thank Y. He and $\mathrm{H}$. Bellen for producing transformants; $\mathrm{E}$. Hafen, H. Bellen, D. Strutt, A. Brand, and the Bloomington Stock Center for fly strains; K. Beckingham for providing fly strains before publication; I. Pass, G. McConnachie, and C.P. Downes for communicating unpublished data; and U. Weber and R. Newsam for technical assistance. D.C.I.G. is grateful to V.R. Goberdhan for her support and advice. This study benefited from the use of the SEQNET facility. The work in C.W.'s laboratory was supported by grants from the Biotechnology and Biological Sciences Research Council and the Wellcome Trust.

The publication costs of this article were defrayed in part by payment of page charges. This article must therefore be hereby marked "advertisement" in accordance with 18 USC section 1734 solely to indicate this fact.

\section{References}

Ashburner, M. 1989. Drosophila: A laboratory handbook. Cold Spring Harbor Laboratory Press, Cold Spring Harbor, NY.

Baker, J., J.P. Liu, E.J. Robertson, and A. Efstratiadis. 1993. Role of insulin-like growth factors in embryonic and postnatal growth. Cell 75: 73-82.

Böhni, R., J. Riesgo-Escovar, S. Oldham, W. Brogiolo, H. Stocker, B.F. Andruss, K. Beckingham, and E. Hafen. 1999. Autonomous control of cell and organ size by CHICO, a Drosophila homolog of vertebrate IRS1-4. Cell 97: 865-875.

Brand, A.H. and N. Perrimon. 1993. Targeted gene expression as a means of altering cell fates and generating dominant phenotypes. Development 118: 401-415.

Capdevila, J. and I. Guerrero. 1994. Targeted expression of the signaling molecule decapentaplegic induces pattern duplications and growth alterations in Drosophila wings. EMBO $\mathrm{J}$. 13: 4459-4468.

Cavener, D.R. 1987. Comparison of the consensus sequence flanking translational start sites in Drosophila and vertebrates. Nucleic Acids Res. 15: 1353-1361.

Chen, C., J. Jack, and R.S. Garofalo. 1996. The Drosophila insulin receptor is required for normal growth. Endocrinology 137: 846-856. 
Cline, T.W. 1978. Two closely linked mutations in Drosophila melanogaster that are lethal to opposite sexes and interact with daughterless. Genetics 90: 683-698.

Di Cristofano, A., B. Pesce, C. Cordon-Cardo, and P.P. Pandolfi. 1998. PTEN is essential for embryonic development and tumour suppression. Nat. Genet. 19: 348-355.

Eaton, S., R. Wepf, and K. Simons. 1996. Roles for Rac1 and Cdc42 in planar polarization and hair outgrowth in the wing of Drosophila. J. Cell Biol. 135: 1277-1289.

Fischer-Vize, J.A. and K.L. Mosley. 1994. marbles mutants: Uncoupling cell determination and nuclear migration in the developing Drosophila eye. Development 120: 2609-2618.

Fristrom, D., M. Wilcox, and J. Fristrom. 1993. The distribution of PS integrins, laminin A and F-actin during key stages in Drosophila wing development. Development 117: 509-523.

Furnari, F.B., H.-J.S. Huang, and W.K. Cavanee. 1998. The phosphoinositol phosphatase activity of PTEN mediates a serum sensitive G1 growth arrest in glioma cells. Cancer Res. 58: 5002-5008.

Gateff, E.A. and B.M. Mechler. 1989. Tumor-suppressor genes of Drosophila melanogaster. CRC Crit. Rev. Oncogen. 1: 221245.

Gu, J., M. Tamura, R. Pankov, E.H.J. Danen, T. Takino, K. Matsumoto, and K.M. Yamada. 1999. Shc and FAK differentially regulate cell motility and directionality modulated by PTEN. J. Cell Biol. 146: 389-403.

Leevers, S.J., D. Weinkove, L.K. MacDougall, E. Hafen, and M.D. Waterfield. 1996. The Drosophila phosphoinositide 3-kinase Dp110 promotes cell growth. EMBO J. 15: 65846594.

Leevers, S.J., B. Vanhaesebroeck, and M.D. Waterfield. 1999. Signalling through phosphoinositide 3-kinases: The lipids take centre stage. Curr. Opin. Cell Biol. 11: 219-225.

Li, D.-M. and H. Sun. 1997. TEP1, encoded by a candidate tumor suppressor locus, is a novel protein tyrosine phosphatase regulated by transforming growth factor $\beta^{1}$. Cancer Res. 57: 2124-2129.

Li, J., C. Yen, D, Liaw, K. Podsypanina, S. Bose, S.I. Wang, J. Puc, C. Miliaresis, L. Rodgers, R. McCombie et al. 1997a. PTEN, a putative protein tyrosine phosphatase gene mutated in human brain, breast, and prostate cancer. Science 275: 1943 1947.

Li, L., B.R. Ernsting, M.J. Wishart, D.L. Lohse, and J.E. Dixon. 1997b. A family of putative tumor suppressors is structurally and functionally conserved in humans and yeast. J. Biol. Chem. 272: 29403-29406.

Lindsley, D.L. and G.G. Zimm. 1992. The genome of Drosophila melanogaster. Academic Press, San Diego, CA.

Liu, J.P., J. Baker, A.S. Perkins, E.J. Robertson, and A. Efstratiadis. 1993. Mice carrying null mutations of the genes encoding insulin-like growth factor 1 (Igf-1) and type 1 IGF receptor (Igf1r). Cell 75: 59-72.

Maehama, T. and J.E. Dixon. 1998. The tumor suppressor, PTEN/MMAC1, dephosphorylates the lipid second messenger, phosphatidylinositol 3,4,5-trisphosphate. J. Biol. Chem. 273: $13375-13378$.

1999. PTEN: A tumour suppressor that functions as a lipid phosphatase. Trends Cell Biol. 9: 125-128.

Marsh, D.J., V. Coulon, K. Lunetta, P. Rocca-Serra, P. Dahia, Z. Zheng, D. Liaw, S. Caron, B. Duboue, A. Lin et al. 1998 Mutation spectrum and genotype-phenotype analyses in Cowden disease and Bannayan-Zonana Syndrome, two hamartoma syndromes with germline PTEN mutation. Hum. Mol. Genet. 7: 507-515.

Mount, S.M. 1982. A catalogue of splice junction sequences. Nucleic Acids Res. 10: 459-472.
Myers, M.P. and N.K. Tonks. 1997. PTEN: Sometimes taking it off can be better than putting it on. Am. J. Hum. Genet. 61: 1234-1238.

Myers, M.P., J.P. Stolarov, C. Eng, J. Li, S.I. Wang, M.H. Wigler, R. Parsons, and N.K. Tonks. 1997. P-TEN, the tumor suppressor from human chromosome 10q23, is a dual-specificity phosphatase. Proc. Natl. Acad. Sci. 94: 9052-9057.

Myers, M.P., I. Pass, I.H. Batty, J. Van der Kaay, J.P. Stolarov, B.A. Hemmings, M.H. Wigler, C.P. Downes, and N.K. Tonks. 1998. The lipid phosphatase activity of PTEN is critical for its tumor suppressor function. Proc. Natl. Acad. Sci. 95: $13513-13518$.

Neufeld, T.P., A.F.A. Delacruz, L.A. Johnston, and B.A. Edgar. 1998. Coordination of growth and cell division in the Drosophila wing. Cell 93: 1183-1193.

Ogg, S. and G. Ruvkun. 1998. The C. elegans PTEN homolog, DAF-18, acts in the insulin receptor-like metabolic signaling pathway. Mol. Cell 2: 887-893.

Quiring, R., U. Walldorf, U. Kloter, and W.J. Gehring. 1994. Homology of the eyeless gene of Drosophila to the Small eye gene in mice and Aniridia in humans. Science 265: 785-789.

Riesgo-Escovar, J.R., M. Jenni, A. Fritz, and E. Hafen. 1996. The Drosophila Jun-amino-terminal kinase is required for cell morphogenesis but not for DJun-dependent cell fate specification in the eye. Genes \& Dev. 10: 2759-2768.

Sluss, H.K., Z. Han, T. Barrett, D.C.I. Goberdhan, C. Wilson, R.J. Davis, and Y.T. Ip. 1996. A JNK signal transduction pathway that mediates morphogenesis and an immune response in Drosophila. Genes \& Dev. 10: 2745-2758 and 11: 541.

Smith, A. and A. Ashworth. 1998. Cancer predisposition: Where's the phosphate? Curr. Biol. 8: R241-R243.

Staehling-Hampton, K. and F.M. Hoffmann. 1994. Ectopic decapentaplegic in the Drosophila midgut alters the expression of five homeotic genes, $d p p$ and wingless, causing specific morphological defects. Dev. Biol. 164: 502-512.

Stambolic, V., A. Suzuki, J.L. de la Pompa, G.M. Brothers, C Mirtsos, T. Sasaki, J. Ruland, J.M. Penninger, D.P. Siderovski, and T.W. Mak. 1998. Negative regulation of PKB/ Akt-dependent cell survival by the tumor suppressor PTEN. Cell 95: 29-39.

Staveley, B.E., L. Ruel, J. Jin, V. Stambolic, F.G. Mastronardi, P. Heitzler, J.R. Woodgett, and A.S. Manoukian. 1998. Genetic analysis of protein kinase B (AKT) in Drosophila. Curr. Biol. 8: 599-602.

Steck, P.A., M.A. Perhouse, S.A. Jasser, A. Yung, H. Lin, A.H. Ligon, L.A. Langford, M.L. Baumgard, T. Hattier, T. Davis et al. 1997. Identification of a candidate tumour suppressor gene, MMAC1, at chromosome 10q23.3 that is mutated in multiple advanced cancers. Nat. Genet. 15: 356-362.

Suzuki, A., J.L. de la Pompa, V. Stambolic, A.J. Elia, T. Sasaki, I.B. Barrantes, A. Ho, A. Wakeham, A. Itie, W. Khoo et al. 1998. High cancer susceptibility and embryonic lethality associated with mutation of the PTEN tumor suppressor gene in mice. Curr. Biol. 8: 1169-1178.

Tamura, M., J. Gu, K. Matsumoto, S. Aota, R. Parsons, and K.M. Yamada. 1998. Inhibition of cell migration, spreading and focal adhesions by tumor suppressor PTEN. Science 280: $1614-1617$

Teng, D.H.-F., R. Hu, H. Lin, T. Davis, D. Iliev, C. Frye, B. Swedlund, K. Hansen, V. Vinson, K. Gumpper et al. 1997. MMAC1/PTEN mutations in primary tumor specimens and tumor cell lines. Cancer Res. 57: 5221-5225.

Thomas, G.H., D.C. Zarnescu, A.E. Juedes, M.A. Bales, A. Londergan, C.C. Korte, and D.P. Kiehart. 1998. Drosophila $\beta_{\text {Heavy }}$-spectrin is essential for development and contributes 


\section{Goberdhan et al.}

to specific cell fates in the eye. Development 125: 21252134.

Toker, A. 1998. The synthesis and cellular roles of phosphatidylinositol 4,5-bisphosphate. Curr. Opin. Cell Biol. 10: 254261.

Treisman, J.E., N. Ito, and G.M. Rubin. 1997. misshapen encodes a protein kinase involved in cell shape control in Drosophila. Gene 186: 119-125.

Wang, S.I., J. Puc, J. Li, J.N. Bruce, P. Cairns, D. Sidransky, and R. Parsons. 1997. Somatic mutations of PTEN in glioblastoma multiforme. Cancer Res. 57: 4183-4186.

Wilson, C., D.C.I. Goberdhan, and H. Steller. 1993. Dror, a potential neurotrophic receptor gene, encodes a Drosophila homolog of the vertebrate Ror family of Trk-related receptor tyrosine kinases. Proc. Natl. Acad. Sci. 90: 7109-7113.

Wolff, T. and D.F. Ready. 1993. Pattern formation in the Drosophila retina. In The development of Drosophila melanogaster (ed. M. Bate and A. Martinez-Arias), pp. 1277-1325. Cold Spring Harbor Laboratory Press, Cold Spring Harbor, NY.

Wu, X., K. Senechal, M.S. Neshat, Y.E. Whang, and C.L. Sawyers. 1998. The PTEN/MMAC1 tumor suppressor phosphatase functions as a negative regulator of the phosphoinositide 3-kinase/Akt pathway. Proc. Natl. Acad. Sci. 95: 1558715591.

$\mathrm{Xu}, \mathrm{T}$. and G.M. Rubin. 1993. Analysis of genetic mosaics in developing and adult Drosophila tissues. Development 117: 1223-1237.

Xu, T., W. Wang, S. Zhang, R.A. Stewart, and W. Yu. 1995. Identifying tumor suppressors in genetic mosaics: The Drosophila lats gene encodes a putative protein kinase. Development 112: 1053-1063. 


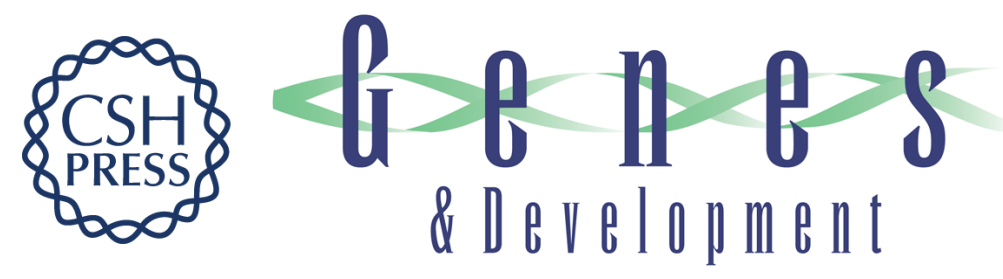

\section{Drosophila tumor suppressor PTEN controls cell size and number by antagonizing the Chico/PI3-kinase signaling pathway}

Deborah C.I. Goberdhan, Nuria Paricio, Emma C. Goodman, et al.

Genes Dev. 1999, 13:

References This article cites 48 articles, 24 of which can be accessed free at:

http://genesdev.cshlp.org/content/13/24/3244.full.html\#ref-list-1

License

Email Alerting Receive free email alerts when new articles cite this article - sign up in the box at the top Service right corner of the article or click here.

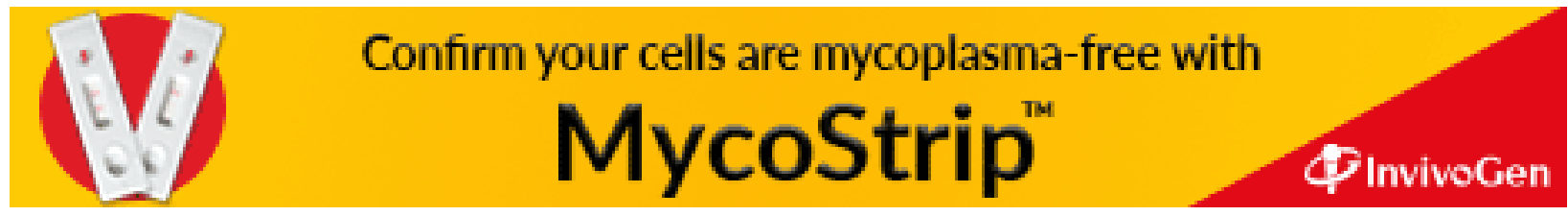

